\title{
Neuronal primary cilia regulate pyramidal cell positioning to the deep and superficial sublayers in the hippocampal CA1
}

\author{
Juan Yang ${ }^{1}$, Liyan Qiu ${ }^{1}$, and Xuanmao Chen ${ }^{1, *}$
}

${ }^{1}$ Department of Molecular, Cellular, and Biomedical Sciences, University of New Hampshire, Durham, NH 03824

${ }^{*}$ Correspondence should be addressed to Dr. Xuanmao Chen:

Department of Molecular, Cellular, and Biomedical Sciences, College of Life Sciences and Agriculture, University of New Hampshire,

389 Rudman Hall, 46 College Road, Durham, NH 03824. Phone: 603862 4542, Fax: 603862 4013, Email:

Xuanmao.Chen@unh.edu

Running Title: Primary Cilia and Pyramidal Cell Positioning

Key Words: Neuronal Primary Cilia, Pyramidal Cell Positioning, Arl13B and AC3, Inside-Out Lamination, and Neuronal Maturation

Acknowledgements: This study was supported by National Institutes of Health Grants K01AG054729, P20GM113131-7006, R15MH126317, and R15MH125305 to X.C.; A UNH CoRE PRP award and Cole Neuroscience and Behavioral Faculty Research Awards to X.C.; UNH Summer TA Research Fellowships (STAF) to J.Y. We are grateful to the University Instrumentation Center at UNH for excellent A1R HD confocal imaging service. We thank the scientists in the Anderson and Gleeson Laboratories who generated the Arl13B-mCherry; Centrin2-GFP double transgenic mouse model. 


\section{Significance Statement}

1. Because the axonemes of primary cilia of early-born neurons point to the stratum oriens (SO), whereas late-born neuronal cilia orient toward the opposite direction, neuronal primary cilia orientation can be used as a marker to distinguish early- and late-born pyramidal neurons in the CA1.

2. This work is the first to show that neuronal primary cilia modulate pyramidal neuronal positioning to the deep and superficial sublayers of the hippocampal CA1.

3. Transgenic overexpression of Arl13B not only elongates primary cilia and promotes earlier cilia protrusion, but also affects centriole positioning and cilia orientation in the CA1.

4. We have identified a novel "reverse movement" of late-born CA1 and cortical pyramidal neurons for cell positioning, which may affect the maturation and formation of synaptic connectivity of late-born neurons. 


\begin{abstract}
It is well-recognized that primary cilia regulate embryonic neurodevelopment, but little is known about their roles in postnatal neurodevelopment. The striatum pyramidal (SP) of hippocampal CA1 consists of superficial and deep sublayers, however, it is not well understood how early- and late-born pyramidal neurons position to two sublayers postnatally. Here we show that the axonemes of primary cilia of earlyborn pyramidal neurons point to the stratum oriens (SO), whereas late-born neuronal cilia orient toward the stratum radiatum (SR), reflecting an inside-out lamination pattern. Neuronal primary cilia in SP undergo marked changes in morphology and orientation from postnatal day 5 (P5) to P14, concurrent with pyramidal cell positioning to the deep and superficial sublayers and with neuronal maturation. Transgenic overexpression of Arl13B, a protein regulating ciliogenesis, not only elongates primary cilia and promotes earlier cilia protrusion, but also affects centriole positioning and cilia orientation in SP. The centrioles of late-born neurons migrate excessively to cluster at SP bottom before primary cilia protrusion and a reverse movement back to the main SP. Similarly, this backward movement of centriole/cilia is also manifested on late-born cortical pyramidal neurons, although early- and late-born cortical neurons display the same cilia orientation. Together, this study provides the first evidence demonstrating that late-born pyramidal neurons exhibit a reverse movement for cell positioning, and primary cilia regulate pyramidal neuronal positioning to the deep and superficial sublayers in the hippocampus.
\end{abstract}




\section{Introduction}

Neuronal primary cilia are hair-like, microtubule-based surface projections, which are identified throughout the nervous system including the hippocampus and cortex (1-3). Neuronal primary cilia are sensitive to diverse extracellular signals, such as hormones and neurotransmitters, and regulate a variety of physiological functions including neurodevelopment, energy balance, and cognitive function (3-6). Defects in neuronal primary cilia lead to various brain disorders including developmental disorders, obesity, and cognitive impairment (4, 6-11). It is well-known that primary cilia modulate embryonic neurodevelopment $(12,13)$ by mediating the Sonic hedgehog $($ Shh) signaling $(3,4,6,14,15)$ and influencing cell fate specification in the ventral neural tube $(16,17)$, forebrain neuronal patterning by regulating radial glia cell polarity (18-20), and proliferation of granule neurons precursors in the cerebellum (21-23). Evidence also supports a role of primary cilia in hippocampus-dependent memory formation in the adult brain. For example, neuronal primary cilia are implicated in hippocampal memory by affecting adult neurogenesis $(13,24,25)$, and forebrain-specific ablation of primary cilia leads to deficits in aversive learning and memory and novel object recognition (26). However, the function of neuronal primary cilia in postnatal neurodevelopment is poorly understood.

Depending on origination or destination, migrating neurons of developing nervous system employ two strategies (27-29): individual or collective migration. Pyramidal neurons in the cortex and hippocampus, which originate from neural tube cells, are individually guided to their destinations by crawling along radial glia fibers (29-31). Individual neuronal migration has three steps for locomotion: leading process extension, nucleokinesis (nuclear translocation), and retraction of trailing process (27). The nuclear translocation of neuronal migration occurs in a saltatory manner, in which the centriole moves forward in the leading process, followed by nuclear translocation that is driven by cytoskeleton dynamics (32-34). The centriole is constantly connected to the nucleus through microtubule network that surrounds the nucleus in a "fork-like" structure (27, 30, 35-37). Hence, centriole and nuclear movements are tightly coupled during the saltatory migration of pyramidal cells. It is worth mentioning that primary cilia possess highly conserved, rigid, $9+0$ doublet axoneme architecture $(38,39)$, which are suited to generate, sense, or engage in cellular mechanical forces (39-45). In this regard, primary cilia, after emanating from centrioles, may mechanically impede saltatory migration during individual neural migration (46).

Accumulating evidence has demonstrated that hippocampal pyramidal neurons exhibit spatially patterned heterogeneity in the adult brain (47-49). Based on the differentiations in molecular, structural, morphological, and physiological features (50-52), the SP of the hippocampus is subdivided into the deep and superficial sublayers along the radial axis $(48,49)$. These two sublayers harbor early- and late-born 
pyramidal neurons, respectively, which migrate along the radial glia axis from the ventricular zone to the hippocampal plate in a birthtime-dependent inside-out pattern $(49,53)$. The deep-superficial lamination of the hippocampus is thought to be crucial for parallel information processing in the hippocampus (49, 53), because neurons with same birthtime in hippocampal sub-regions (CA1, CA3, and dentate gyrus) connect preferentially with each other and exhibit similar patterns of gene expression $(49,54)$. The insideout lamination in the Ammon's horn of the hippocampus shares some similarity to that of the neocortex but has its own features $(49,50)$. While numerous studies have contributed to unveiling the cellular and molecular basis of neuronal migration during embryonic development for cortical lamination $(30,32,53$, 55-57), it remains elusive how hippocampal pyramidal cells position to the deep and superficial sublayers of the hippocampus during early postnatal development.

Neuronal primary cilia can be marked by two ciliary proteins, type 3 adenylyl cyclase (AC3) and ADP-ribosylation factor-like 13B (Arl13B) (2). AC3 is abundantly expressed in neuronal primary cilia in the adult brain $(1,2,58)$. In contrast, Ar113B generally marks neuronal primary cilia in the developing nervous system $(2,59-61)$ and non-neural cells $(2,62)$. Arl13B is an atypical small GTPase that regulates ciliogenesis and the Shh pathway (63-67). While Arl13B plays multifaceted roles during embryonic development including regulating interneuron migration (61), it is unclear if Arl13B affects postnatal neurodevelopment and pyramidal cell positioning in the hippocampus. To determine if primary cilia regulate pyramidal cell positioning to the sublayers of hippocampus, we examined the morphological dynamics and orientation changes of neuronal primary cilia in the hippocampus and neocortex using AC3 and Arl13B antibodies (2). To confirm cilia orientation, we used Arl13B-mCherry; Centrin2-GFP double transgenic mice (Arl13B+ mice) (68), in which mCherry labels Arl13B-positive primary cilia, and GFP marks centrioles via fusion with Centrin2, a centriole marker (69). Because Arl13B promotes ciliogenesis $(65,67,70)$, this transgenic mouse model also allowed for Arl13B gain-of-function studies to examine the effect of longer primary cilia on cell positioning and centriole migration in the hippocampus during postnatal development. We found that primary cilia in SP undergo marked changes in morphology and orientation from P5-P14, concurrent with the formation of synaptic connectivity in the hippocampal CA1 (71-74). Likely due to delayed ciliation, the centrioles of late-born neurons migrate excessively to pass early-born neurons and condense at SP bottom before primary cilia protrusion and a reverse movement back to the main SP. This backward movement for cell positioning is different from typical saltatory migration for nucleokinesis during embryonic development. Our data support the conclusions that lateborn pyramidal cells undergo a reverse movement during early postnatal development, and neuronal primary cilia regulate pyramidal cell positioning to the deep and superficial layers in the hippocampus. 


\section{Materials and Methods}

\section{Mice}

We used WT mice with a C57BL/6j genetic background. The Arl13B-mCherry; Centrin2-GFP mice were purchased from the Jackson Laboratory (Stock No. 027957) and originally had C57BL/6, FVB/N, and BALB/c mixed genetic background. The expression of Arl13B-mCherry and Centrin2-GFP are both driven by the pCAGGs promoter $(65,68,69)$. After importing to the lab, we have backcrossed this strain with C57BL/6j mice for more than six generations to obtain uniform C57BL/6j background. The backcrossing has also yielded three separate mouse lines: one keeps as Arl13b-mCherry; Centrin2-GFP double transgenic (Arl13B + mice), which were used to mark both primary cilia and centrioles; the second becomes Arl13b-mCherry single transgenic mice, which were used for Arl13B gain-of-function studies; the third is Centrin2-GFP single transgenic mice, which labeled centrioles and were served as Control mice for Arl13B + mice, when we investigated if Arl13B affects cilia orientation and centriole positioning. Date were collected from both male and female animals. Mice were maintained on a 12-hour light/dark cycle at $22{ }^{\circ} \mathrm{C}$ and had access to food and water ad libitum. All animal procedures were approved by the Institutional Animal Care and Use Committee at the University of New Hampshire (UNH).

\section{Brain Tissue Collection}

Mice were deeply anesthetized with an intraperitoneal injection of ketamine and xylazine, and then were transcardially perfused with ice-cold $0.1 \mathrm{M}$ phosphate buffer saline (PBS) followed by 4\% paraformaldehyde (PFA) in 0.1 M PBS. Young pups (P0 - P7) were directly decapitated using a sharp surgical scissors. Mouse brains were extracted and then placed into $4 \%$ PFA overnight at $4{ }^{\circ} \mathrm{C}$. The following day, brains were rinsed in $0.1 \mathrm{M}$ PBS for $30 \mathrm{~min}$ at room temperature. P0-P5 mouse brains then were dehydrated by $30 \%$ sucrose in $0.1 \mathrm{M}$ PBS overnight at $4^{\circ} \mathrm{C}$. Finally, those tissue were embedded in O.C.T resin before being sectioned at $-18{ }^{\circ} \mathrm{C}$ using a cryostat to a thickness of $30 \mu \mathrm{m}$. P7 - P75 mouse brains were coronal sectioned using Compresstome (Precisionary Instruments LLC, NC) to a thickness of $40-50 \mu \mathrm{m}$.

\section{Immunofluorescence Staining}

P0-P5 mouse brains were sliced and mounted on gelatin-coated slices for immunostaining procedure, while P7 - P75 brain sections were sliced and placed in 24-wells plate, staying afloat during the staining procedure. They were washed three times with 0.1 M PBS. Then they were washed in $0.2 \%$ Triton X-100 (vol/vol) in 0.1 M PBS for three times for 10 minutes at room temperature. Then they were blocked in 
blocking buffer (0.1M PBS containing 10\% normal donkey serum (vol/vol), 2\% bovine serum albumin (weight/vol), $0.2 \%$ Triton $\mathrm{X}-100(\mathrm{vol} / \mathrm{vol})$ ) for one hour at room temperature. After that, slices were incubated with primary antibodies overnight at $4{ }^{\circ} \mathrm{C}$ in blocking buffer. After washing in $0.1 \mathrm{M}$ PBS for three times for 10 minutes, slices were incubated with secondary antibodies for 2 hours at room temperature. Primary antibodies were rabbit anti-AC3 (1:10000, EnCor Biotechnology Inc., \#RPCAACIII), mouse anti-Ar113B (1:500, UC Davis clone N295B/66), mouse anti-calbindin (1:1000, Swant Switzerland, D-28K), and mouse anti-NeuN (1:500, Millipore-Sigma, MAB377, clone A60). Secondary anti-bodies were Alexa Fluor 488-, 546-, or 647-conjugated (Invitrogen, La Jolla, CA). Finally, sections were counter-stained with DAPI (Southern Biotech, \#OB010020) and images were acquired with confocal microscope (Nikon, A1R-HD), installed in the University Instrument Center at UNH.

\section{Cilia Orientation Measurement}

When a single primary cilium in WT mice was measured, the relative thinner axoneme was defined as the tip and the opposite site as the base. For Ar113B-mCherry \& Centrin2-GFP transgenic mice, the location of the primary cilium base was the same as Centrin2-GFP. The tails of most primary cilia in WT mice were straight and easy to determine their angles. However, primary cilia in the Arl13B-mCherry \& Centrin2-GFP transgenic mice were much longer and showed kinks in their axoneme (65). For those primary cilia, the initial part ( $\sim 2 \mu \mathrm{m}$ from the base) was used for determining the angle of those primary cilia. Fiji was utilized to measure the orientation of the single primary cilium. Four steps were used to complete a measurement. 1) First, the angle tool was selected among icons showed in the main window. 2) Then, the shift button on the keyboard was held, while left clicking the mouse meanwhile to draw a line (always from the right to left and parallel to the external capsule) to the location of the base of the primary cilium. 3) Afterwards, the shift button was released, and the mouse was left clicked to draw a line from the base to the tip of the primary cilium. 4) Lastly, while holding the control button and selecting "M" on the keyboard, the angle would jump out in a separate window. To measure the next primary cilium, steps 2-4 were repeated. Acquired data were finally exported as an excel file and then to a MATLAB program, which was used to generate polar plots.

\section{Primary Cilia Length Measurement}

We measured the length of primary cilia based on published methods (75), with modifications. The freehand line tool within Fiji (ImageJ) was used to measure cilia length from the base of axoneme to the distal tip. 40- $\mu \mathrm{m}$ Z-stack confocal images in Fig. 2 were used for both of adult WT and Arl13B+ mice. 20- 
$\mu \mathrm{m}$ Z-stack confocal images were used for Fig. 1, Fig. 4, P3, P7, P10, and P14 Arl13B+ mice. Confocal images of $20 \mu \mathrm{m}$ Z-stack were used for Fig. 7.

\section{CA1 SP Thickness Estimation}

Confocal images of $20 \mu \mathrm{m} \mathrm{Z-stack} \mathrm{were} \mathrm{used} \mathrm{for} \mathrm{quantifying} \mathrm{CA1} \mathrm{SP} \mathrm{thickness} \mathrm{for} \mathrm{both} \mathrm{WT,} \mathrm{Arl13B+}$ mice and Control mice. The straight line tool within Fiji was used to measure CA1 SP thickness from the top of SP to the bottom of SP based on nucleus staining (76). Because the nuclei at the top and bottom edge of SP are not aligned to a straight line, this causes some variation of SP thickness. For young adult mice, we chose coronal sections at $-1.9 \mathrm{~mm}$ posterior to Bregma to measure CA1 SP thickness. For young pups, we measured the thickness based on selected slices with hippocampal anatomic geometry similar to adult hippocampal sections. We acknowledge that our estimation method has limitations and stereotaxic accuracy needs to be improved. Nevertheless, it is known (76) that CA1 SP thickness decreases markedly with age, particularly during the first two weeks. We present CA1 SP thickness changes with ages to demonstrate that hippocampal pyramidal cells maintain certain movement or re-positioning postnatally, yielding significant changes during early postnatal period (before P14) and mild adjustment afterwards.

\section{Quantification of nucleus density, centriole density, and ratio of centriole to nucleus (C/N Ratio)}

A $70 \mu \mathrm{m} \times 70 \mu \mathrm{m}$ square box in SP was used for one single area quantification. The freehand line tool within Fiji was utilized to draw a line that matched the margin of the nucleus at the bottom of SP in the chosen box. This line was then vertically moved down $15 \mu \mathrm{m}$ (to the SR direction) and up $15 \mu \mathrm{m}$ (to the SO direction). The panel between two lines was defined as the bottom panel of SP for quantifying nucleus, centrioles, and $\mathrm{C} / \mathrm{N}$ Ratio. The top line at the bottom panel of SP was further moved up $30 \mu \mathrm{m}$ (to the SO direction). The area between the two lines (having a width of $30 \mu \mathrm{m}$ ) was defined as the middle panel of $\mathrm{SP}$ for the quantification of nuclei and centrioles. Since many centrioles were found to be clustered in the SR particularly in AR113B+ mice, we also included a $15-\mu \mathrm{m}$-width area in the SR that was next to SP for quantification (Fig. 5I). Two Centrin2 dots were counted as one pair of centrioles, one DAPI separate round region were counted as one nucleus.

\section{Statistical and Software}

Data were analyzed with Fiji, GraphPad Prism and MATLAB. Data was considered as statistically significant if $\mathrm{p}<0.05$ and values in the graph are expressed as mean \pm standard error of the mean. Statistical analyses were conducted using ANOVA for multiple group comparison or Student's T-test with a two-tailed distribution, as appropriate. N.S., Not Significant; ${ }^{*} \mathrm{p}<0.05 ; * * \mathrm{p}<0.01 ; * * * \mathrm{p}<0.001$. 


\section{Results}

Neuronal primary cilia of early- and late-born hippocampal pyramidal cells exhibit opposite orientation.

The SP of hippocampal CA1 consists of deep- and superficial-sublayer, inhabited by early- and late-born pyramidal cells, respectively $(47-49,55)$. It is not well understood how early- and late-born pyramidal cells position themselves into two sublayers postnatally. We performed immunofluorescence staining using AC3 and Arl13B antibodies to examine the morphological changes of neuronal primary cilia in SP during postnatal development. We found that primary cilia orientation in SP undergoes marked changes during early postnatal development (Fig. 1A-D). At P7, AC3- or Arl13B-positive neuronal primary cilia were rarely detected in hippocampal SP of WT mice (Fig. 1A). At P10, neuronal primary cilia protruded and became prominent, with many clustered at the edge of SP and SR (Fig. 1A). Interestingly, they exhibited opposite orientation: the axoneme of primary cilia at the bottom (superficial sublayer) mostly pointed in the SR direction, whereas those at the top (deep sublayer) oriented to the SO direction. From $\mathrm{P} 10$ to $\mathrm{P} 14$, cilia orientation underwent a drastic change, $40 \%$ of cilia pointed to SO (north) at P10, while at P14 nearly $20 \%$ of cilia pointed to SO (Fig. 1D). At P14, superficial-sublayer neuronal cilia occupied the major space of the SP, and the axonemes of primary cilia almost uniformly pointed in the SR direction. Deep-sublayer neurons were then squeezed to a thin layer at the top and their cilia kept an orientation towards SO. Afterwards (e.g., at P40 and P75), cilia orientation did not change much (Fig. 1C-D), except for becoming less uniformly in line with the radial axis (Fig. 1A). However, primary cilia of superficialsublayer neurons were still aligned with the radial axis and oriented toward SR. Polar plots and histogram plots present changes in cilia orientation from P10 to P75 (Fig. 1C). It is noteworthy that the laminar thickness of SP decreased significantly from P3 to P14 and reached a steady-state someday between P14 and P40 (Fig. 1E-F), suggesting that pyramidal cells continue to adjust their position postnatally, which condenses cell density and decreases CA1 lamina thickness.

To confirm if primary cilia with opposite orientation come from early- and late-born pyramidal neurons, respectively, we performed immunofluorescence staining using an antibody of calbindin, which is a marker for late-born neurons in the dorsal hippocampal CA1 after P20 (49, 77). Fig. $1 \mathrm{G}$ shows that primary cilia of calbindin-positive neurons in the superficial sublayer largely point in the direction of SR, whereas calbindin-negative neurons' primary cilia generally orient toward the SO direction, supporting the notion that early- and late-born pyramidal neurons exhibit opposite cilia orientations. To demonstrate early- and late-born neuron distribution in SP, we include in situ hybridization (ISH) results of Col11a1 and Htrla adopted from Allen Brain Atlas (https://mouse.brain-map.org/). Coll1al is a marker of early- 
born pyramidal neurons (48), while Htrla is selectively expressed in late-born neurons (49). ISH results show that Coll1al mRNA is mostly detected in the deep sublayer, with a small fraction also distributed to the superficial sublayer (Fig. 1H). Htrla mRNA is identified largely in the superficial sublayer, whose staining signals do not cover all areas of the superficial sublayer (Fig. 1I). This suggests that although early- and late-born pyramidal neurons markedly differentiate in function and morphology and inhabit the deep- and superficial-sublayers, respectively, there is no clear bound to physically separate two groups of neurons in CA1 sublayers. Together, these data indicate that primary cilia of early- and late-born hippocampal pyramidal neurons exhibit opposite orientation, suggesting a role of neuronal primary cilia in pyramidal cell positioning during early postnatal development.

\section{Arl13B regulates neuronal ciliogenesis, cilia orientation, and pyramidal cells positioning to CA1 sublayers.}

We also observed that neuronal cilia marker AC3 expression in pyramidal cells in the CA1 remains relatively stable after P10 (Fig. 1A)(2). In contrast, Ar113B started to emerge at P7 with staining identified strongly in SP bottom (Fig. 1A top). At P10, Arl13B expression reached a very high level in the SP, particularly strong at SP bottom, then decreased at P14 and further reduced at P40, and became very weak in adulthood (Fig. 1A). Arl13B high expression appears to occur within the same time-window as pyramidal cell positioning and neuronal maturation (the development of synaptic connectivity) from $\sim$ P5 to $\mathrm{P} 14$ (71-73, 78), suggesting a role in neuronal positioning. Arl13B is a ciliary small GTPase that modulates ciliogenesis, interneuron migration and maturation $(20,66,67)$. We have previously reported that Arl13B+ mice have much longer neuronal primary cilia in the hippocampus (65). Although Arl13B+ mice display a high percentage of seizure phenotype partly due to their hybrid FVB background (65), we overcame this limitation by backcrossing this strain with $\mathrm{C} 57 \mathrm{Bl} / 6 \mathrm{~J}$ mice for over six generations. It turned out that $\mathrm{Arl13B}+$ mice in $\mathrm{C} 57 \mathrm{Bl} / 6 \mathrm{~J}$ background do not manifest seizure phenotypes. We found that neuronal primary cilia are the longest in the hippocampal CA1, compared to several other brain regions including CA3, dentate gyrus, cortex, and amygdala in adults (Fig. 2). Moreover, we compared the cilia length of Arl13B+ adult mice with WT adult mice and found that the effect of Arl13B on elongating cilia length was prominent in the CA1, and much weaker in other regions including amygdala and cortex (Fig. 2). This highlights an important role of neuronal primary cilia in the CA1 and Arl13B in regulating neuronal cilia length.

To determine if Arl13B overexpression affects cilia orientation, we next characterized changes in cilia orientation during postnatal development using Arl13B + mice. First, we found that at P7 neuronal 
primary cilia were prominent in Arl13B+ mice (Fig. 3A), which was different from WT mice (Fig. 1A). Deep-sublayer neuronal primary cilia largely pointed to the SO direction, whereas primary cilia of superficial-sublayer neurons clearly show opposite cilia orientation (Fig. 3A). Second, immunostaining images from $\mathrm{P} 7$ to $\sim \mathrm{P} 75$, polar and histogram plots of cilia orientation indicate that primary cilia of Arl13B + mice underwent drastic changes in orientation from P7 to P14 and mild changes from P14 to P75 (Fig. 3A-C), similar to WT mice. This suggests that the time-window before P14 is crucial for the changes in cilia orientation. Third, overexpression of Arl13B affected the percentage of primary cilia having SO or SR orientation: at P14 and P40, more primary cilia pointed to the north in Arl13B+ mice than WT mice (Fig. 3D). Note that at P7 neuronal primary cilia in WT mice were not well expressed, but Arl13B + mice have a large fraction of cilia pointing to the north (Fig. 3A-C). Fourth, the lamina thickness of CA1 SP decreased significantly from P3 to P14, and further at $\sim$ P75 (Fig. 3E-F), suggesting that CA1 neurons continuously adjust their position. Lastly, we also used calbindin staining to verify that primary cilia with opposite orientation were indeed from early- and late-born pyramidal neurons, respectively (Fig. $3 \mathrm{G}-\mathrm{H})$. These data demonstrate significant changes in cilia orientation and strong effects of Arl13B in modulating CA1 cilia orientation during early postnatal development. In addition, neuronal primary cilia orientation can be used as a marker to distinguish early- and late-born neurons in the CA1 region.

Drastic changes in cilia morphology and orientation were found before P14 in both WT and Arl13B+ mice (Fig. 1\&2), which was concurrent with the high expression of Arl13B in the CA1 (Fig. 1A) and with neuronal positioning time-window. This suggests a role of Arl13B in pyramidal cell positioning by modulating ciliogenesis. To determine if Arl13B affects ciliation timing and pyramidal cell positioning to deep- and superficial sublayers of SP, next we examined changes in cilia morphology and orientation as well as centriole distribution in the CA1 from P3 to P14. We found that overexpression of Arl13B not only elongated primary cilia, but also affected the timing of ciliation (Fig. 4A). Arl13B+ mice started to express primary cilia in the deep sublayer as early as P3, all of which pointed to SO (Fig. 4A). At P5, more primary cilia emerged from deep-sublayer neurons, and some from superficial-sublayer neurons in the SP bottom (Fig. 4A). At P7, primary cilia of deep-sublayer neurons became very long, while primary cilia of superficial-sublayer neurons were not fully elongated, with many remained short or dot-shaped at the bottom of SP (Fig. 4A). We quantified the cilia length of deep- and superficial sublayer at P3, P7, P10, and P14. Clearly, the cilia length of deep-sublayer neurons was much longer than that of superficialsublayer neurons from P3 to P10, but at P14 both groups of neuronal cilia reached a plateau level (Fig. 4B). These data demonstrate that the expression of primary cilia of deep-sublayer (early-born) neurons is earlier than superficial-sublayer (late-born) neurons in Arl13B+ mice. In contrast, while WT early-born 
neurons protrude a few SO-pointing cilia as early as P3 and P5, primary cilia were barely detectable at P7 in the superficial layers in the CA1 (Fig. 4A). AC3 staining become a bit more obvious in the deep sublayer than superficial sublayer at P7. At P9, WT mice started to express many short cilia in the main SP, and at P10 primary cilia become longer, however, these cilia distribution did not display a deep-superficial sublayer distinction as clearly as that of Ar113B+ mice at P7, P9, or P10 (Fig. 4A). These images indicate that Arl13B promotes earlier primary cilia protrusion and affects cilia distribution in the CA1.

The results that primary cilia of early-born neurons emerge earlier than that of late-born neurons and both reach full length at P14 raised the questions if early-born pyramidal neurons mature earlier than late-born pyramidal neurons and if neuronal maturation and ciliation occur within the same time-window. To examine if there is a birthtime-dependent maturation in SP sublayers, we employed an antibody of NeuN, a protein marker for matured pyramidal neurons $(79,80)$, in our immunostaining assay. Fig. 4C shows that the expression of NeuN at P1 was not detected, except for some weak staining in the deep sublayer of SP. At P5, NeuN expression became stronger in the deep sublayer, but not much in the superficial sublayer. At P7, the superficial sublayer started to have higher NeuN expression, and at P14, NeuN was abundantly expressed in both deep- and superficial-sublayers. This "first deep - then superficial" sequential NeuN expression pattern indicates an order of birthtime-dependent neuronal maturation in the CA1. To determine if glutamatergic synaptic markers (indicative of synaptic connectivity) show a similar pattern, we searched Allen Brain Atlas Developing Mouse Brain (https://developingmouse.brainmap.org/). Indeed, we found that many synaptic marker proteins of glutamatergic synapses including Shank3 (Fig. 4D), and Homer-1 (Fig. 4E), as well as Camk2a, PSD-95, Synapsin-1 and Syntaxin 1 (Table 1, ISH data available in the Atlas), display a similar sequential expression pattern: first expressed in the deep sublayer of SP at P4 or so, then extended to the superficial sublayer and reached full expression at P14 (Fig. 4D-E). This expression sequence is in accordance with the order of primary cilia protrusion from P5 to P14 first in the deep and then superficial sublayer of the CA1 in Arl13B+ mice.

We acknowledge that WT mice have dot-shaped primary cilia in the superficial sublayer of SP at P7 (Fig. 4A). Nevertheless, it is known that several steps of ciliogenesis occur under the plasma membrane and before cilia protrusion $(81,82)$. In addition, we did detect SO-pointing primary cilia in the deep sublayer as early as P3 and P5 in WT mice (Fig. 4A), indicating that neuronal ciliogenesis does occur in CA1 deep sublayer of WT mice before P9. Arl13B overexpression promotes earlier cilia protrusion and amplifies cilia expression, which allowed us to visualize hair-like primary cilia and reveal this new ciliation pattern. Interestingly, the timing of ciliation and neuronal maturation is not solely dictated by neuron's birthtime. For example, CA3 neurons are born around the same time as CA1 neurons and CA3 
pyramidal layer also follows an inside-out lamination pattern (55), although there are other types of neurons migrating to CA3 (50). We found that the hippocampal CA3 of WT mice had abundant expression of primary cilia at P5 (not shown) and P7 in both deep and superficial sublayers (Fig. 4F), whereas the CA1 did not (Fig. 4A), indicating that CA3 protrudes neuronal primary cilia several days earlier than CA1. Consistently, hippocampal CA3 pyramidal neurons mature several days earlier than CA1 neurons, as evidenced by NeuN expression pattern and strong expression of several glutamatergic synapse marker proteins including Camk2a and PSD-95 in the CA3 at P4 (Table 1), in contrast to very weak and deep sublayer-restricted expression in the CA1 (Table 1 and Fig. 4G). ISH data of other glutamatergic synapse markers also exhibit similar patterns (Table 1 and ISH data from https://developingmouse.brain-map.org/). In addition, the deep-superficial distinctions in ciliation and maturation in CA3 are much weaker than CA1 (Fig. 4). These data support the argument that hippocampal neuronal ciliation is closely related to neuronal maturation, not strictly to birthtime. Together, we conclude that neuronal ciliation in CA1 sublayers coincides with neuronal and synaptic maturation in an order of "first deep - then superficial".

\section{After being clustered at SP bottom, centrioles/cilia are subjected to a reverse movement to position late-born pyramidal cells to the superficial sublayer of CA1 SP.}

We further observed that cilia density in the main SP of Arl13B+ mice at P7 was very low, and obviously there were more nuclei than primary cilia (Fig. 4A). A large fraction of cilia/centrioles was instead congregated at SP bottom, and not many cilia were found in the middle panel of SP (Fig. 4A). However, a few days later at P14, cilia distribution became more even in SP (Fig. 3A). This suggests that the centrioles of late-born pyramidal neurons migrate excessively to reach and cluster at SP bottom before redistribution.

To test it, we quantified and compared nucleus density, centriole density, and the ratio of centriole to nucleus (C/N Ratio) in the CA1 SP at P3, P7, P10 and P14 using both Arl13B+ and Control mice (Fig. 5A-B). Comparison of Arl13B+ with Control mice also enabled us to unveil the effects of Arl13B overexpression on centriole positioning. For better quantification, we artificially separated confocal images of SP into top, middle, and bottom panels (as shown in Fig. 5I). We also included a section of SR (15 $\mu \mathrm{m}$ width) in the quantification because some centrioles also reached SR (Fig. 5I). First, we discovered that Arl13B+ and Control mice do not show significant differences in nucleus density at the middle and bottom panels from P3 to P14 (Fig. 5A-B), indicating that neurons' nuclei are evenly distributed in the SP during this period. However, centrioles were highly condensed at SP bottom at P3 and P7 in both Arl13B+ and Control mice (Fig. 5A-B), because the C/N Ratio in SP bottom and SR were much higher than 1 (Fig. 
5D). Second, centriole density at the bottom panel was much higher than at middle panel at P3 and P7 in both Arl13B+ and Control mice (Fig. 5C). However, subsequently at P10 and P14, centrioles show a relatively even distribution in the SP (Fig. 5A\&C). Consistently, C/N Ratio in both Arl13B+ and Control mice at P3 were about 2.5, but it gradually decreased to around 1 at P14 (Fig. 5D). Third, Arl13B+ mice have a lower centriole density at the middle panel than Controls at P3 and P7 (Fig. 5C), but their C/N Ratio in SP bottom and SR were much higher than Control mice at P7 (Fig. 5D), indicating a higher enrichment of centrioles in SP bottom in Arl13B+ mice than Control mice. This result also demonstrates an effect of Arl13B on centriole positioning or distribution in the SP.

The changes in centriole distribution from P3 to P14 also suggest that there was a reverse movement (from SP bottom back to main SP) for centriole positioning. To verify this, we took and presented confocal images of both Centrin2 and Arl13B. Indeed, at P7 in Arl13B+ mice, cilia density in the upper part of SP was very low, while centrioles and short primary cilia were highly condensed at SP bottom (Fig. 5E). At P10, neuronal primary cilia became much longer. Some centrioles/cilia became wellaligned with radial glia fiber, while others still clustered at SP bottom (Fig. 5E), suggesting that some neurons started to move toward the north (SO direction), and centrioles took the lead, followed by hairlike cilia tail (Fig. 5E). At P14, long cilia occupied the major space of SP, and their primary cilia almost uniformly pointed to the SR direction (parallel to the radial glia fiber). There were no more centrioles/cilia clustering at SP bottom (Fig. 5E). These data indicate that centriole and cilia move reversely to redistribute from the bottom of SP to the main SP. We quantified cilia orientation of the upper part of SP. Consistently, the upper part of SP originally only had SO-pointing cilia at P7. Due to this "pull-back" cilia/centriole movement, more south-oriented cilia gradually moved to main SP from P7 to P14, the upper part of SP subsequently contained more south-oriented cilia (Fig. 5G-H). Together, we conclude that the cilia/centrioles of late-born pyramidal neurons move against the saltatory migration direction to reposition to the superficial sublayer. We name this phenomenon "reverse movement", as contrast to typical forward saltatory migration. A schematic model in Fig. 5F depicts the cilia orientations and the direction of the reverse movement. These data also suggest that the reverse movement of late-born neurons from P7 to P14 may indirectly affect late-born neurons' maturation or their postnatal development of synaptic connectivity, which occurs several days later than early-born neurons in the CA1 (Fig. 4C-E).

\section{Late-born cortical pyramidal neurons also undergo a reverse movement for cell positioning and protrude primary cilia later than early-born neurons.}


Hippocampal CA1 and the neocortex share similarities in their neuronal migration mode in the embryonic stage $(30,50,83)$. For instance, they consist of the same originated early-born and late-born pyramidal neurons, which inhabit the deep- and superficial sublayer, respectively (49). Next, we asked if postnatally primary cilia of cortical pyramidal neurons exhibit similar pattern to that of hippocampal CA1. We found that in terms of cilia orientation, neuronal primary cilia in cortical Layer II/III (superficial sublayer) most pointed to the pial surface both at P14 and P40 (Fig. 6A), consistent with results in the CA1 superficial sublayer and with previous report (84). However, late-born pyramidal neurons in cortical Layer V (deep sublayer) did not exhibit opposite cilia orientation to early-born neurons (Fig. 6A). Instead, most of primary cilia of Layer V neurons still pointed to the pial surface, although Layer II neurons had a higher percentage of cilia pointing to the pial surface (Fig. 6B). Cortical Layer VI does not harbor many pyramidal neurons and their cilia orientation was different from Layer II/III and Layer V (Fig. 6C). These data indicate that early- and late-born cortical pyramidal neurons have the same cilia orientation, which is different from that of CA1.

To determine if cortical pyramidal neurons are subjected to reverse movement as late-born hippocampal neurons before P14, we examined centriole/nuclei distribution pattern in the superficial sublayer (Layer II/III) of the neocortex at P3 and P14 (Fig. 7A). For better quantification, we drew top and middle panels on the images of Layer II/III to quantify cilia and nuclei density. We found that the density of centrioles (Fig.7B) and nuclei (Fig.7C) at the top panel were higher than those at the middle panel at P3. However, at P14 both centriole and nuclei density between two panels had no significant differences. These data indicate that the densities of centrioles and nuclei in Layer II/III have changed from compact and uneven at P3 to sparse and even at P14. This strongly suggests that there is a reverse movement from the top to middle panel, which distributes centriole/nuclei more evenly at P14 (Fig. 7BC) and makes neuronal cilia pointing to the pial surface (Fig. 6A). However, the $\mathrm{C} / \mathrm{N}$ ratios were near 1 and there were no significant differences between P3 and P14 and between the top and middle panels (Fig. 7D), suggesting that there is no excessive clustering of centrioles at Layer II/III of the neocortex, which is different from the case of hippocampal CA1.

To determine if cilia expression of cortical neurons similarly exhibit an order of "first deep - then superficial", we compared the ciliation timing of early- and late-born cortical neurons. We found that at P3 and P7 primary cilia of early-born cortical neurons were much longer than those of late-born neurons (Fig. 7E-H), indicating that early-born cortical neurons express primary cilia earlier than late-born neurons. Consistently, ISH results from Allen Brain Atlas also demonstrate that Shank3 and Homer1 display similar sequential pattern in the neocortex: first expression in the cortical deep sublayer at $\mathrm{P} 4$, then extended to 
the superficial sublayer at P14 (Fig 4D-E). Other synaptic markers, such as PSD-95 and syntaxin-1, also exhibit similar patterns (Table 1) (https://developingmouse.brain-map.org/). Therefore, this maturation sequence of pyramidal neurons in cortical sublayers is similar to that of hippocampal CA1, supporting the notion that neuronal ciliation is related to cell positioning, reverse movement, and neuronal maturation in both hippocampal and cortical sublayers.

\section{Discussion}

This study provides the first evidence demonstrating that neuronal primary cilia play an important role in postnatal neurodevelopment by modulating pyramidal cell positioning to the deep and superficial sublayers. (1) The axoneme of primary cilia of early-born neurons point to SO, whereas those of late-born neurons display opposite cilia orientation. Neuronal primary cilia in the hippocampal SP undergo drastic changes in orientation and morphology from P5-P14, concurrent with pyramidal cell positioning to the deep and superficial sublayers and with neuronal maturation. (2) Transgenic overexpression of Arl13B not only elongates primary cilia and promotes earlier cilia protrusion, but also affects centriole migration and cilia orientation in the CA1 SP. (3) We identified a novel migration mode of centrioles/cilia of lateborn neurons for cell positioning, which is different from typical saltatory migration for nucleokinesis: After reaching SP bottom, centrioles of late-born neurons protrude primary cilia and then undergo a "reverse movement" back to the main SP from P7-P14, which makes the cilia axoneme of late born neurons point to SR. (4) The ciliation of pyramidal neurons in the neocortex also occurs within neuronal positioning time-window, but neuronal primary cilia in the deep and superficial sublayers exhibit the same orientation. (5) After migrating to and congregating in Layer II/III of the neocortex at P3-P7, late-born pyramidal neurons protrude primary cilia, followed by a reverse movement to re-distribute neurons in the superficial sublayer. The reverse movement helps explain why neuronal primary cilia in Layer II/III point to the pial surface.

\section{P5-P14 is a critical developmental time-window for neuron ciliation, pyramidal cell positioning to}

\section{CA1 sublayers, and neuronal maturation.}

It has been documented that pyramidal neurons in the mouse hippocampus are generated during embryonic days (E) 12 - 18 (85, 86), and their generation peaks between E14 and E16 (83, 85, 87). After early- and late-born pyramidal neurons migrate from the ventricular zone or sub-ventricular zone to the hippocampal plate, early-born neurons position to the deep sublayer of SP and late-born neurons migrate to pass early-born neurons and reside in the superficial sublayer $(50,88)$. This is so called birthdate- 
dependent inside-out lamination pattern $(49,55)$. Although embryonic developmental profiling of earlyand late-born neurons have been well characterized (47-49,88), how those neurons postnatally adjust their final positions in the CA1 sublayers and settle down to develop stable synaptic connectivity are poorly understood. It is known that excitatory synaptic connectivity in the mouse hippocampus develop and neuronal become matured in the first two weeks of life (71-74), however, it is unknown why CA1 pyramidal neurons need two weeks to construct stable synapses, and if neuronal primary cilia engage in neuronal positioning and maturation process. Our data demonstrate that $\sim \mathrm{P} 5$ to P14 is a critical period for early- and late-born pyramidal neurons to position to the deep- and superficial sublayers in the CA1, respectively. First, primary cilia undergo drastic changes in morphology and orientation from P5-P14 (Fig. 1A, 2A, 3A). The thickness of SP lamina decreases significantly from P3 to P14, concurrent with cilia protrusion and marked changes in cilia orientation (Fig. 1D and 3C). Second, Arl13B+ mice start to express primary cilia first from early-born neurons at P3 or before. Several days later at P7, late-born CA1 neurons of Arl13B+ mice started to express primary cilia. After P14, both cilia length and orientation do not change much, reaching a steady state. WT mice exhibit similar pattern and their early-born hippocampal neurons also express SO-pointing primary cilia at P3-P7, albeit much fewer (Fig. 4A). In addition, centriole distribution in SP changes from clustering at SP bottom at P3-P7 to even distribution in the main SP at P14 (Fig. 5A-D). Together, cilia length, cilia orientation, CA1 lamina thickness, and centriole/cilia distribution in the SP are all subjected to marked changes from P5-P14 and reach a plateau at P14. These changes additionally show an order of "first deep - then superficial". Similarly, neuronal maturation also shows a similar pattern. We show that NeuN, a marker for matured neurons (79), starts to brightly express only in early-born neurons in SP at P5, followed by late-born neurons at P7, and reach abundant expression in both early- and late-born neurons at P14 (Fig. 4C). Consistently, many synaptic markers of excitatory neurons including Shank3 (Fig. 4D) and Homer1 (Fig. 4E), as well as Camk2a, PSD-95 (Fig. 4G), Synapsin-1, and Syntaxin 1 (Table 1, ISH data from the Allen Brain Atlas Developing Mouse Brain), first emerge from early-born neurons in the CA1 around P4, then extend to late-born neurons, reaching a plateau in both early- and late-born neuron at P14. These data indicate that early-born CA1 pyramidal neurons mature earlier than late-born neurons.

Interestingly, CA3 pyramidal neurons mature several days earlier than CA1 pyramidal neurons (Fig. 4G), as evidenced by the expression of synaptic marker proteins, including Camk2a, PSD-95, Synapsin-1, and Syntaxin 1 in the Allen Brain Atlas (summarized in Table 1). We have discovered that CA3 protrude hair-like primary cilia earlier than CA1 in WT mice (Fig. 4F), supporting the notion that primary cilia expression is closely related to neuronal maturation. These data underscore the interesting 
question why late-born CA1 pyramidal neurons need to wait until P14 to become matured. We reason this is most likely because the reverse movement of CA1 late-born neurons occurs from P7 to P14 (Fig. 5E). We show that the CA1 lamina thickness can remarkably reduce from $90 \mu \mathrm{m}$ to $\sim 70 \mu \mathrm{m}$ from P7 to P14 (Fig. 1) and reach a steady-state level of $\sim 60 \mu \mathrm{m}$, afterwards CA1 SP thickness is only subject to very mild changes, so does cilia orientation (Fig. 1\&3). Because late-born neurons undergo a striking reverse movement (over a distance of $10 \mu \mathrm{m}$ to $30 \mu \mathrm{m}$ ) from P7 to P14, it could destabilize the cytoskeleton of dendrites and synapses, affecting the formation and stability of synaptic connections. This reasoning is consistent with numerous reports showing that CA1 glutamatergic synapses before P14 are either silent or labile, and their electrophysiological features are different from mature synapses after P14 (71-73, 89, 90). Likewise, cortical Layer II/III late-born neurons also mature later than Layer V early-born neurons (Fig 7D-E) (ISH data of many synaptic markers available in the Allen Brain Atlas Developing Mouse Brain). Our data indicate that late-born cortical neurons also have a reverse movement before P14 (Fig. 7A), and their late maturation could be, at least in part, explained by the reverse movement. Collectively, this study on cilia orientation and mRNA expression analyses of Allen Brain Atlas demonstrate that $\sim$ P5 to P14 represents the critical period to position early- and late-born neurons to the deep- and superficialsublayer of hippocampal CA1, respectively. The reverse movement of late-born neurons likely delays their final cell positioning, neuronal maturation, or formation of synaptic connectivity in the superficial sublayer. These discoveries are of high significance and raise many interesting questions that warrant future studies. Clearly revealing cell positioning pattern in the forebrain will help understand how the pyramidal cell heterogeneity of two sublayers enables parallel information processing in neural networks, affecting learning and memory formation.

\section{Pyramidal cell positioning to the deep and superficial sublayers is regulated by centriole positioning, ciliation timing, and cilia orientation.}

While pyramidal neurons in the hippocampal CA1 and neocortex are arranged in an inside-out layered structure, cortical lamina volume expand significantly during postnatal neurodevelopment (91), but the CA1 SP is gradually condensed in the first two weeks. Although studies have demonstrated that the lamina thickness difference can be partially explained by different migration mode in the embryonic stage (49), it is not very clear how the SP is further subdivided into the superficial and deep sublayers in a very thin laminar structure.

This study has examined the dynamics of cilia morphology and orientation in superficial and deep sublayers during postnatal development. We found that early-born neurons protrude primary cilia earlier 
than late-born neurons and have longer cilia at P3-P7 in the CA1 and neocortex (Fig. 4A-B, 7E-H). The ciliation timing and cilia length difference in two sublayers is consistent with the expression pattern of NeuN staining and synapses makers (Fig. 4C-E). This suggests that ciliation timing is closely related to neuronal maturation in the deep and superficial sublayers. Moreover, we have identified an opposite cilia orientation in pyramidal neurons in the deep and superficial sublayers of CA1 SP (Fig 1A and 3A). In contrast, neuronal primary cilia in both deep and superficial sublayers of the neocortex exhibit the same orientation (Fig. 6A) and aligns with apical dendrite of cortical pyramidal neurons (Fig. 3A). It is possible that not only the superficial sublayer but also the deep sublayer in the cortex is subjected to the same direction of reverse movement for neuronal positioning during postnatal development, which could consequently expand the lamina volume of neocortex. Nevertheless, more evidence is needed to support such an interpretation.

Neuronal primary cilia are oriented oppositely in early- and late- born neurons to promote the separation of the deep- and superficial-sublayer in the CA1 SP. In WT mice, due to cilia shortness, it is difficult to dissect cilia expression and orientation pattern in the deep and superficial layers. Arl13B, which is highly enriched in primary cilia during early neurodevelopment (Fig. 1), drastically elongates cilia length in CA1 pyramidal neurons in Arl13B + mice (Fig. 2). The Arl13B + model enabled us to better observe the effects of neuronal primary cilia on pyramidal cell positioning. We clearly show that earlyborn neurons neuronal cilia expression several days earlier than late-born neurons, which may impact the moving dynamic of neuronal migration and cause centriole distribution variation (Fig. 5A-D). We reason that the ciliation timing is critical for the modulating effect of primary cilia on cell positioning. Arl13B overexpression promotes the early expression of primary cilia of early-born neurons, which may impede centriole migration of early-born neurons toward the SR direction. Consequently, this enhances the separation of centrioles/cilia of early-born neurons with those of late-born neurons (Fig. 5).

The centriole/centrosome, as the microtubule-organizing center, directs cellular microtubule dynamics that couple to nuclear translocation during individual cell migration $(30,33,34,92)$. Polarized centriole positioning is found to be important for properly oriented cell division, polarized cilia positioning, and cell migration (93). Our findings suggest that centriole positioning and ciliation timing affects the pyramidal neuron positioning to the deep and superficial sublayers of SP (Fig. 5F). After early- and lateborn pyramidal neurons reach the CA1 SP, their centrioles exhibit distinctions. The centrioles of earlyborn neurons do not migrate excessively or separate from their nuclei distantly. They position themselves in the proximity of nuclei. Primary cilia then protrude out of early-born neurons and point towards SO (Fig.1-4), which is opposite to the pyramidal neuron saltatory migration direction (from SO to SR). This 
rigid surface protrusion may slow down (or terminate) the saltatory migration of early-born neurons and help stabilize them in the deep sublayer. On the other hand, the expression of primary cilia of late-born neurons occurs several days later than early-born neurons (Fig. 4). Likely due to delayed cilia protrusion (other factors cannot be excluded), centrioles of late-born neurons excessively migrate a long distance, distant themselves from their nuclei. They reach and congregate at SP bottom, where they start to protrude primary cilia. Afterwards, centrioles/cilia of late-born neurons undergo a reverse movement back to the main SP (Fig. 5E). The differences in centriole positioning, ciliation timing, and cilia orientation promotes the sorting of early- and late-born neurons to the deep and superficial sublayers, respectively. Apparently not only the cilia/centrioles but also the cell body of late-born neurons move backwards, because the lamina thickness of CA1 SP decreases significantly from P3 to P14. Early-born neurons likely complete cell positioning and become matured before P7, while late-born neurons are still experiencing a reverse movement toward SO from P7-P14. Early- and late-born neuronal cilia point in the opposite direction, suggesting that primary cilia are likely to serve as mechanical anchors for adjusting cell positioning and movement. It is not uncommon for primary cilia to engage in mechanical forces (39-45). For example, a recent study has shown that mechanical strain controls the length, motility, and planar position of primary cilia, affecting the determination of left-right body axis during gastrulation (94). We also have shown that olfactory cilia can sense mechanical force of airflow to regulate olfactory perception (95). Given unique mechanical features of primary cilia, primary cilia protrusion may function like "mechanical anchor of centrioles" to regulate pyramidal cell positioning to the deep and superficial sublayers. Nevertheless, besides mechanical implication of primary cilia, some planar cell polarity molecules, which are yet to be identified, may also direct the positioning of centrioles and cilia expression of CA1 pyramidal cells.

This study has revealed several interesting distinctions between early- and late-born pyramidal neurons in the hippocampal CA1: early-born neurons' centrioles migrate a shorter distance and position in the proximity of nuclei; they express primary cilia earlier, which point to SO; they complete cell positioning earlier, their synapses mature earlier; and they do not experience a reverse movement. In contrast, late-born pyramidal neurons migrate a longer distance; Their centrioles reach and cluster at SP bottom, which is distal to the nuclei; they express primary cilia several days later, which point to SR; afterwards late-born neurons are subjected to a reverse movement; they complete cell positioning several days later, and consequently late-born neurons (and their synaptic connectivity) mature several days later.

In summary, this report presents the first and critical evidence demonstrating that neuronal primary cilia regulate pyramidal cell positioning to CA1 sublayers. We also document that the reverse movement of late-born pyramidal neurons from the bottom of SP back to the main SP is important for establishing 
the deep-superficial layered structure in the hippocampal CA1. The reverse movement may indirectly affect the maturation or formation of synaptic connectivity of late-born neurons.

\section{Figure Legends}

Fig. 1. Opposite primary cilia orientation in the deep and superficial sublayers of hippocampal SP. $(\boldsymbol{A}-\boldsymbol{C})$ Opposite orientation of primary cilia in the hippocampal SP of WT mice. (A) Coimmunofluorescence staining using AC3 (red) and Arl13B antibody (green) on hippocampal slices from P7 to $\sim$ P75. Cilia orientations exhibit marked change with ages from P10 to $\sim$ P75. Scale bars: $10 \mu \mathrm{m}$. (B) A diagram of cilia orientation measurement in the dorsal hippocampal CA1. Cilia are posited in the polar plot on the right. Polar plots analyses were performed to determine primary cilia orientation distribution. The numbers $\left(0-360^{\circ}\right)$ on the out circle denote the degree of cilia orientation relative to the external capsule, and the numbers on concentric circles are the percentage of cilia orientation at specific angles. $(\boldsymbol{C})$ Polar plots and histogram plots showing cilia orientation pattern and their corresponding percentages. Most primary cilia axoneme point to the SR direction $\left(180^{\circ}-360^{\circ}\right)$ in polar plots (Left). Histogram plots (binned in 10-degree intervals, Right) presenting the percentage of cilia at different angles. The degrees in polar plots were converted to histogram plots by subtracting $180^{\circ}$. (D-F) Changes of cilia orientation coincide with the decrease of SP thickness during postnatal development. (D) Statistical data showing the percentage of north-oriented cilia (pointing to SO direction). The percentage of north-oriented cilia decreased significantly from P10 to P14. A $100 \mu \mathrm{m}$ x $100 \mu \mathrm{m}$ square is standardized area for quantifying total cilia in the SP. 3 areas for each mouse, total 9 areas from 3 mice. Each triangle represents one area in SP for quantification, N.S., not-significant, *** $\mathrm{p}<0.001$; one-way ANOVA with Bonferroni's multiple comparison test. $(\boldsymbol{E})$ The thickness of SP lamina in the hippocampal CA1 decreases significantly from P3, P5, P10, P14, to P40 and P75. 9 locations for one mouse, 3 mice for each age, mix sex. Each triangle represents one location in SP for quantification, N.S., not-significant, *** $\mathrm{p}<0.001$; one-way ANOVA with Bonferroni's multiple comparison test. (F) Co-immunostaining of AC3 (red) with Arl13B (green) and DAPI (blue) on brain slices at P3, P14, and $\sim$ P75. Primary cilia orientation changed from well-aligned (P14) to less ordered ( P75). Scale bars: $20 \mu \mathrm{m}$. (G) Immunofluorescence staining using AC3 and calbindin antibodies. Primary cilia of calbindin-positive neurons at P40 mostly pointed in the SR direction, while calbindin-negative neurons' cilia generally point in the SO direction. The white boxes are enlarged. Cilia basal body is bigger than axoneme, allowing for determining cilia orientation. (H-I) Allen Brain Atlas in situ hybridization staining of Col11a1 and Htrla in mouse hippocampal CA1 at P56. Col11a1 is mostly expressed in the deep sublayer, but also slightly dispersed to the superficial sublayer $(\boldsymbol{H})$. Htrla is 
mostly present in the superficial sublayer and covers most, but not all area of SP (I). Images credit: Allen Institute for Brain Science. Super: superficial sublayer; Deep, deep sublayer. P7: Postnatal Day 7. SP: stratum pyramidale; SO: stratum oriens; SR: stratum radiatum. Images credit: Allen Institute for Brain Science

Fig. 2. Strong regulation of CA1 neuronal cilia length by Arl13B. (A-B) Transgenic Arl13B mice $(\mathrm{Arl13B}+)$ have much longer neuronal primary cilia than WT mice in the hippocampal subregions (CA1, CA3 and DG) in adults. Notably, The CA1 region has the longest primary cilia and the effect of Arl13B on promoting CA1 cilia length is prominent. Compared with WT mice, the average CA1 cilia length increases by over $60 \%$ in Arl13B + mice. (C-D) Primary cilia length is not strongly changed in other brain regions such as cortex and amygdale. Immunostaining of neuronal primary cilia in WT controls and Arl13B overexpression transgenic $(A r 113 B+)$ mice. Unpaired Student's T-test was applied in $(\boldsymbol{B}$ and $\boldsymbol{D})$, and significance is indicated by $* * *, p<0.001$. N.S., not-significant; Scale bar: $10 \mu \mathrm{m}$. Data were collected from at least 3 young adult mice for each genotype. Mix sex.

Fig. 3. Opposite primary cilia orientation and marked cilia orientation changes in the hippocampal SP of Arl13B+ mice. (A-C) Deep- and superficial-sublayer neurons in AR113B+ mice display opposite primary cilia orientation. (A) Postnatal cilia orientation changes at P7, P10, P14, P40, and P75. White boxes are enlarged at the bottom. White arrows indicate the mother centriole (basal body), yellow arrows denote some centrin2-GFP artifacts. After P14, green artifacts caused by Centrin2-GFP expression became prominent $(65,69)$, but Centrin2-GFP was still able to indicate the basal body of primary cilia. Scale bars: $10 \mu \mathrm{m}$. (B) Polar plots and histogram plots of cilia orientation of Arl13B + mice. Cilia orientation is opposite, and the majority of primary cilia point to SR (to the south). (C) Comparison of the percentage of north-oriented cilia at different ages of Arl13B + mice. The percentage of north-oriented cilia decreases significantly from P7, P10, to P14, and reaches a plateau after P14. 3 areas for each mouse, total 9 areas for 3 mice. Mix sex. Each triangle represents the percentage of north-oriented cilia from an area of CA1 SP for quantifying cilia orientation. N.S., not significant, *** $\mathrm{p}<0.001, * * \mathrm{p}<0.01$; oneway ANOVA with Bonferroni's multiple comparison test. (D) Comparison of north-oriented cilia percentage between Ar113B+ and Control mice (Centrin2-GFP single transgenic mice) at different ages. Arl13B+ mice have more north-oriented primary cilia in CA1 SP than Control mice at P7, at P14 and P40. $(\boldsymbol{E}-\boldsymbol{F})$ The thickness of CA1 SP lamina in Arl13B+ mice also decreases with age postnatally. 9 locations for one mouse, 3 mice for each age. Mix sex. Each triangle represents one location in SP for quantification. 
N.S., not significant, $* * * \mathrm{p}<0.001$; one-way ANOVA with Bonferroni's multiple comparison test. Primary cilia of Arl13B + mice are well aligned with radial axis at P14 (F). (G-H) Immunofluorescence staining using AC3 and calbindin antibodies on hippocampal slices from Arl13B+ mice and Control mice. Scale bars: $10 \mu \mathrm{m}$. (G) Primary cilia of calbindin-positive neurons mostly point in the direction of SR (white arrows), while those of calbindin-negative neurons generally point to SO (yellow arrows). (H) Statistical data of Ar113B+ and WT mice. Over 80\% cilia in the calbindin-positive region (superficial sublayer) point in the SR direction and less than 30\% cilia in the calbindin-negative region (deep sublayer) point in the SR direction. SP: stratum pyramidale; SO: stratum oriens; SR: stratum radiatum. $\mathrm{n}=3$ mice, 3 regions in the CA1 of each mouse were selected for quantification. Exception: for P14 and P40 of Control mice, $\mathrm{n}=2$. Mix sex.

\section{Fig. 4. Early-born CA1 pyramidal neurons protrude primary cilia and mature earlier than late-}

born neurons. $(\boldsymbol{A})$ Left: WT late-born neurons do not express many hair-like primary cilia until P9. At P5, only early-born neurons express north-pointing cilia. At P7, hair-like cilia are hard to be seen in the CA1 and AC3 immunostaining signal is only detected at centrosomes (92), with limited enrichment at SP bottom. At P9, primary cilia are expressed throughout CA1 but very short. At P10, primary cilia show opposite orientation in SP, and most cilia in superficial sublayer point in the direction of SR (Fig. 1B). Right: Earlier and stronger expression of primary cilia in Arl13B+ mice. Arl13B+ mice at P3 start to protrude primary cilia and at P5 more cilia emerge, which are mostly from early-born neurons and pointed to SO. At P7, both early-born neurons and late-born neurons express primary cilia. Cilia of early-born neurons clearly point to SO, whereas cilia of late-born neurons point to SR. Many centrioles/cilia are highly condensed at SP bottom. At P7, P9, and P10, primary cilia exhibit a clear deep-superficial distinction. ( $\left.\boldsymbol{A}^{\prime}\right)$ At P3, a few primary cilia are expressed in early-born CA1 neurons in both WT and Arl13B + mice and they point to SO. Centrioles in Arl13B+ mice were marked by Centrin2-GFP. Data were collected from at least 3 mice each group. Scale bars: $10 \mu \mathrm{m}$. (B) Primary cilia first emerge from deep-sublayer neurons, then from superficial-sublayer neurons. Primary cilia length of deep- and superficial-sublayer neurons of Ar113B+ mice at P3, P7, P10, and P14. At P14, cilia length reaches the steady-state and cilia length has no difference between two sublayers. Over 280 total cilia were quantified in each subject. Unpaired Student's T-test; ${ }^{* * *} \mathrm{p}<0.001$, N.S., not significant. $(\boldsymbol{C})$ NeuN expression was first detected weakly in deep-layer CA1 neurons at P1, more at P5, then extended to superficial-layer neurons P7. At P14, both deep- and superficial-layer neurons had abundant NeuN expression. Scale bars: $10 \mu \mathrm{m}$. (D-E) Allen Brain Atlas in situ hybridization (ISH) results of Shank3 and Homer1, two synaptic 
makers of excitatory neurons, in hippocampal CA1 at P4 and P14. At P4, Shank3 and Homer1 are only expressed in deep sublayer in hippocampal SP and Layer V (deep sublayer) of the neocortex. At P14, ISH signals of both genes occupy most area of SP and the six layers in the neocortex. Scale bars: $100 \mu \mathrm{m}$. Super: superficial sublayer; Deep, deep sublayer. (F) The hippocampal CA3 region of WT mice abundantly express primary cilia at P5 (not shown) and P7, earlier than the CA1. Scale bars: $10 \mu \mathrm{m}$. (G) CA3 pyramidal neurons mature earlier than CA1 neurons. At P4, CamK2a and PSD-95, two postsynaptic marker proteins, are highly and widely expressed throughout the CA3. In contrast, their expression is only restricted to the deep sublayer of CA1, not present in the superficial sublayer at P4. Scale bars: $100 \mu \mathrm{m}$. ISH images were adopted from the Allen Brain Atlas Developing Mouse Brain. Images credit: Allen Institute for Brain Science.

\section{Fig. 5. Centrioles/cilia of late-born neurons undergo a reverse movement after migrating to and} being clustered at SP bottom. (A-D) Centrioles are clustered at SP bottom from P3 to P7 and then redistribute back to main SP. (A) Both Arl13B+ mice (Arl13B-mCherry; Centrin2-GFP mice) and Control mice (Centrin2-GFP littermates) showed a clustering pattern of centrioles in SP bottom from P3 to P7, and then re-distributed back to main SP at P10. Scale bars: $10 \mu \mathrm{m}$. (B) Quantification of the number of nuclei per square millimeter (Nuclei/mm^2) in the middle and bottom panels of SP from P3 to P14. The middle and bottom panel of SP as well as SR are defined in (I) and Methods Section. B: bottom; M: middle. (C) Quantification of centriole number per square millimeter (centrioles $/ \mathrm{mm}^{\wedge} 2$ ) in the middle and bottom panel of SP from P3 to P14. B: bottom; M: middle. (D) The ratio of centriole to nucleus $(\mathrm{C} / \mathrm{N}$ Ratio) at SP bottom and SR from P3 to P14. C/N Ratio data were collected from the bottom panel of SP in combination with SR panel (See I). (E) Centriole/cilia of late-born neurons exhibit a reverse movement after being clustered at SP bottom. Centrioles/cilia had dramatic changes in orientation and length at SP bottom from P7 to P14. Enlarged cilia images of late-born neurons show that cilia length at the superficial sublayer of SP was increasing, and cilia and centrioles were migrating upward (from SR to SO) from P7 to P14. Centrioles (arrows) in the front were followed by cilia axoneme. The P7 image is from Figure 3A. Scale bars: $10 \mu \mathrm{m}$. (F) A schematic model depicting the reverse movement of centrioles/cilia of late-born neurons in the dorsal hippocampus for cell positioning. Late-born neurons move against the saltatory migration direction back to main SP. It is unclear if early-born neurons, with opposite cilia orientation, have forward movement during this period. (G) Polar plots of cilia orientation in the upper part of SP from P7 to P14. Upper part: 70\% top part of SP. Primary cilia in the upper part of SP at P7 largely pointed in the direction of SO (to the north). However, from P7 to P14, many south-oriented primary cilia moved 
back to main SP, consequently changing the cilia orientation in the upper part of SP. $(\boldsymbol{H})$ The percentage of south-oriented cilia in the upper part of SP significantly increases from P7 to P14, confirming that lateborn neurons continuously moved back to occupy the main SP during that period. N.S., not significant, *** $\mathrm{p}<0.001$; one-way ANOVA with Bonferroni's multiple comparison test. P: postnatal. Number: days. We selected 3 CA1 regions each mouse for quantification. $n=3$. Mix sex. Exception: P14 Control, $n=2$, mix sex. (I) An example showing how the middle and bottom panel of SP as well as SR were defined and used for quantification, and more details are found in the "Materials and Methods". Scale bars: $10 \mu \mathrm{m}$.

Fig. 6. Primary cilia in both superficial and deep sublayers of the neocortex point to the pial surface. $(\boldsymbol{A}-\boldsymbol{C})$ Cilia orientation pattern in Layer II/III (superficial sublayer) and Layer V (deep sublayer) in the neocortex. (A) Left, immunostaining results of the superficial sublayer and deep sublayer in the neocortex from P14 and P40 from Arl13B+ mice. Scale bars: $10 \mu \mathrm{m}$. Right: Cilia orientation was quantified and summarized by polar plots (method details for polar plots can be found in Fig. $1 B-C$ ). Most primary cilia perpendicularly pointed to the pial surface in both superficial and deep sublayers of the neocortex. (B) Statistical data showing that over $70 \%$ of cilia point to the north (the pial surface) in both superficial and deep sublayer in the neocortex, but superficial sublayer has higher percentage of north-oriented cilia than deep sublayer. 3 different regions selected for both superficial and deep sublayer in the cortex of each mouse for quantification. N.S., not significant, $* * \mathrm{P}<0.01, * * * \mathrm{P}<0.001$, two-way ANOVA with Bonferroni posttests. $\mathrm{n}=3$. Mix sex. (C) A schematic of cilia orientation generally in sublayers of the neocortex. Data for cilia orientation in Layer VI were collected, but not shown here. Layer I does not have many pyramidal neurons, and we have not examined neuronal cilia in Layer IV.

Fig. 7. Late-born cortical pyramidal cells also exhibit a reverse movement before P14 and protrude cilia later than early-born neurons. (A-D) The densities of centrioles and nuclei in the superficial sublayer (Layer II/III) of the neocortex changed from compact and uneven at P3 to sparse and even at P14. Confocal images of Layer II/III at P3 and P14 of Arl13B + mice (A). WT mice exhibited a similar distribution pattern (not shown). The top and middle panels (as depicted) of superficial sublayer (Layer II/III) are enlarged on the right. Scale bars: $10 \mu \mathrm{m}$. The density of centrioles $(\boldsymbol{B})$ and nuclei $(\boldsymbol{C})$ at top and middle panels. Note that the density of centrioles and nuclei at P3 are higher than those of P14, which could be in part due to an increase in brain and cell body volume with age. (D) The ratios of centriole to nucleus $(\mathrm{C} / \mathrm{N}$ ratio) in both top and bottom of the cortical superficial sublayer at P3 and P14 are all close to 1 , means that no centrosomes clustered phenomenon in the superficial sublayer in the cortex from P3- 
P14. 3 different regions selected for both top and middle of the superficial sublayer in the cortex of each mouse for quantification in $(\boldsymbol{B}-\boldsymbol{D})$. Two-way ANOVA test with Bonferroni posttests was applied in $(\boldsymbol{B}$ $\boldsymbol{D})$, and significance is indicated by $* * * \mathrm{p}<0.001$, N.S., not significant. (E-H) Early-born cortical neurons express primary cilia earlier than late-born neurons. $(\boldsymbol{E}-\boldsymbol{G})$ Co-immunostaining of AC3, Arl13B and DAPI in Layer II and V on brain slices at P3 and P7 from WT mice $(\boldsymbol{E})$ and Ar113B+ mice $(\boldsymbol{G})$. Scale bars: 5 $\mu \mathrm{m}$. $(\boldsymbol{F}, \boldsymbol{H})$ Quantification of cilia length in cortical Layer II and V. Primary cilia of early-born neurons are much longer than those of late-born neurons at P3 and P7 in both WT $(\boldsymbol{F})$ and Arl13B+ mice $(\boldsymbol{H})$. Over 150 cilia were quantified for $(\boldsymbol{F}-\boldsymbol{H}) .3$ different regions selected in both cortical Layer II and V in each mouse for cilia length quantification. Unpaired Student's T-test; *** $\mathrm{p}<0.001 . \mathrm{n}=3$ mice, mix sex for each group for $(\boldsymbol{B}-\boldsymbol{D}, \boldsymbol{F}-\boldsymbol{H})$.

Table 1. Features of neuronal primary cilia and neuronal maturation in subregions of the hippocampus and neocortex

\begin{tabular}{|c|c|c|c|c|c|c|c|c|}
\hline Regions & $\begin{array}{l}\text { CA1 } \\
\text {-deep }\end{array}$ & $\begin{array}{c}\text { CA1 } \\
\text {-super. }\end{array}$ & $\begin{array}{l}\text { Cortex } \\
\text {-deep }\end{array}$ & $\begin{array}{l}\text { Cortex } \\
\text {-super. }\end{array}$ & $\begin{array}{l}\text { CA3 } \\
\text {-deep }\end{array}$ & $\begin{array}{c}\text { CA3 } \\
\text {-super. }\end{array}$ & DG-out ${ }^{\mathbb{1}}$ & DG-in ${ }^{\pi}$ \\
\hline $\begin{array}{l}\text { Ciliation Time } \\
\text { (WT) }\end{array}$ & P3-P7 & P9-P10 & P3-P7 & P7-P10 & P3-P7 & P3-P7 & P3-P7 & After P7 \\
\hline $\begin{array}{l}\text { Ciliation Time } \\
(\text { Arl13B }+)\end{array}$ & $\mathrm{P} 0-\mathrm{P} 7$ & P7-P10 & $\mathrm{P} 0-\mathrm{P} 7$ & P7-P10 & $\mathrm{P} 0-\mathrm{P} 7$ & $\mathrm{P} 0-\mathrm{P} 7$ & $\mathrm{P} 0-\mathrm{P} 7$ & After P7 \\
\hline $\begin{array}{c}\text { Cilia } \\
\text { Orientation }\end{array}$ & $\begin{array}{l}\text { Point to } \\
\text { SO }\end{array}$ & $\begin{array}{l}\text { Point to } \\
\text { SR }\end{array}$ & $\begin{array}{l}\text { Point to } \\
\text { pial surface }\end{array}$ & $\begin{array}{l}\text { Point to } \\
\text { pial surface }\end{array}$ & $\begin{array}{c}\text { Largely }^{\dagger} \\
\text { point to SO }\end{array}$ & $\begin{array}{c}\text { Largely }^{\dagger} \\
\text { point to SR }\end{array}$ & ND & $\begin{array}{c}\text { Point to } \\
\text { outer layer? }\end{array}$ \\
\hline \multirow{2}{*}{$\begin{array}{l}\text { Cilia Length } \\
\text { (WT) }\end{array}$} & $<1 \mu \mathrm{m}, \mathrm{P} 7$ & $<1 \mu \mathrm{m}, \mathrm{P} 7$ & $3.2 \mu \mathrm{m}, \mathrm{P} 7$ & $1.0 \mu \mathrm{m}, \mathrm{P} 7$ & $2.8 \mu \mathrm{m}, \mathrm{P} 7$ & $3.5 \mu \mathrm{m}, \mathrm{P} 7$ & $2.7 \mu \mathrm{m}, \mathrm{P} 7$ & $1.7 \mu \mathrm{m}, \mathrm{P} 7$ \\
\hline & $\begin{array}{l}7.0 \mu \mathrm{m}, \\
\text { Adult }\end{array}$ & $\begin{array}{l}7.0 \mu \mathrm{m}, \\
\text { Adult }\end{array}$ & $\begin{array}{l}6.1 \mu \mathrm{m}, \\
\text { Adult }\end{array}$ & $\begin{array}{l}5.0 \mu \mathrm{m}, \\
\text { Adult }\end{array}$ & $\begin{array}{l}5.6 \mu \mathrm{m}, \\
\text { Adult }\end{array}$ & $\begin{array}{l}5.6 \mu \mathrm{m}, \\
\text { Adult }\end{array}$ & $\begin{array}{l}2.3 \mu \mathrm{m}, \\
\text { Adult }\end{array}$ & $\begin{array}{l}2.3 \mu \mathrm{m} \\
\text { Adult }\end{array}$ \\
\hline \multirow{3}{*}{$\begin{array}{l}\text { Cilia Length } \\
(\text { Arl13B }+)\end{array}$} & $1.5 \mu \mathrm{m}, \mathrm{P} 3$ & $1 \mu \mathrm{m}, \mathrm{P} 3$ & $2 \mu \mathrm{m}, \mathrm{P} 3$ & $0.6 \mu \mathrm{m}, \mathrm{P} 3$ & \multirow{3}{*}{$\begin{array}{l}8.2 \mu \mathrm{m}, \\
\text { Adult }\end{array}$} & \multirow{3}{*}{$\begin{array}{l}8.2 \mu \mathrm{m}, \\
\text { Adult }\end{array}$} & \multirow{3}{*}{$\begin{array}{l}3.1 \mu \mathrm{m}, \\
\text { Adult }\end{array}$} & \multirow{3}{*}{$\begin{array}{l}3.1 \mu \mathrm{m}, \\
\text { Adult }\end{array}$} \\
\hline & $4.5 \mu \mathrm{m}, \mathrm{P} 7$ & $3 \mu \mathrm{m}, \mathrm{P} 7$ & $4.6 \mu \mathrm{m}, \mathrm{P} 7$ & $2 \mu \mathrm{m}, \mathrm{P} 7$ & & & & \\
\hline & $\begin{array}{l}11.9 \mu \mathrm{m}, \\
\text { Adult }\end{array}$ & $\begin{array}{l}12.0 \mu \mathrm{m}, \\
\text { Adult }\end{array}$ & $\begin{array}{l}6.3 \mu \mathrm{m}, \\
\text { Adult }\end{array}$ & $\begin{array}{l}5.3 \mu \mathrm{m}, \\
\text { Adult }\end{array}$ & & & & \\
\hline $\begin{array}{c}\text { Show Reverse } \\
\text { Movement }\end{array}$ & No & $\begin{array}{c}\text { Yes } \\
\text { (P7-P14) }\end{array}$ & Yes? & $\begin{array}{c}\text { Yes } \\
\text { (P3-P14) }\end{array}$ & No & Yes? & ND & ND \\
\hline $\begin{array}{c}\text { NeuN\# } \\
\text { Expression }\end{array}$ & P3-P5 & P7-P14 & $\mathrm{P} 1$ & $\mathrm{P} 4-\mathrm{P} 5^{f}$ & P3-P5 & P3-P5 & P5-P7 & After P7 \\
\hline $\begin{array}{l}\text { Camk2a* } \\
\text { Expression }\end{array}$ & $\sim \mathrm{P} 4$ & $\begin{array}{c}\text { After P4 } \\
\text { before P14 }\end{array}$ & $\sim \mathrm{P} 4$ & $\begin{array}{c}\text { After P4 } \\
\text { before P14 }\end{array}$ & $\sim \mathrm{E} 18.5-\mathrm{P} 4$ & $\sim \mathrm{E} 18.5-\mathrm{P} 4$ & $\sim \mathrm{P} 4$ & After P4 \\
\hline $\begin{array}{l}\text { Shank3* } \\
\text { Expression }\end{array}$ & $\sim \mathrm{P} 4$ & $\begin{array}{c}\text { After P4 } \\
\text { before P14 }\end{array}$ & $\sim \mathrm{P} 4$ & $\begin{array}{c}\text { After P4 } \\
\text { before P14 }\end{array}$ & $\sim \mathrm{E} 18.5-\mathrm{P} 4$ & Unclear & $\sim \mathrm{P} 4$ & After P4 \\
\hline $\begin{array}{l}\text { Homer } 1^{*} \\
\text { Expression }\end{array}$ & $\sim \mathrm{P} 4$ & $\begin{array}{c}\text { After P4 } \\
\text { before P14 }\end{array}$ & $\sim \mathrm{P} 4$ & $\begin{array}{c}\text { After P4 } \\
\text { before P14 }\end{array}$ & $\sim \mathrm{P} 4-\mathrm{P} 14$ & $\sim \mathrm{P} 4-\mathrm{P} 14$ & $\sim \mathrm{P} 4$ & After P4 \\
\hline $\begin{array}{l}\text { PSD-95* } \\
\text { Expression }\end{array}$ & $\begin{array}{l}\sim \mathrm{P} 4 \text { or } \\
\text { earlier }\end{array}$ & $\begin{array}{c}\text { After P4 } \\
\text { before P14 }\end{array}$ & $\sim \mathrm{P} 4$ or earlier & $\begin{array}{c}\text { After P4 } \\
\text { before P14 }\end{array}$ & $\sim \mathrm{E} 18.5-\mathrm{P} 4$ & $\sim \mathrm{E} 18.5-\mathrm{P} 4$ & $\sim \mathrm{P} 4$ & After P4 \\
\hline $\begin{array}{l}\text { Synapsin } 1^{*} \\
\text { Expression }\end{array}$ & $\sim \mathrm{P} 4$ & $\begin{array}{c}\text { After P4 } \\
\text { before P14 }\end{array}$ & $\sim \mathrm{P} 4$ & $\begin{array}{c}\text { After P4 } \\
\text { before P14 }\end{array}$ & $\sim$ E18.5-P4 & $\sim \mathrm{E} 18.5-\mathrm{P} 4$ & $\sim \mathrm{P} 4$ & After P4 \\
\hline $\begin{array}{l}\text { Syntaxin1* } \\
\text { Expression }\end{array}$ & $\sim \mathrm{P} 4$ & $\begin{array}{c}\text { After P4 } \\
\text { before P14 }\end{array}$ & $\sim \mathrm{P} 4$ or earlier & $\begin{array}{c}\text { After P4 } \\
\text { before P14 }\end{array}$ & $\sim \mathrm{E} 18.5-\mathrm{P} 4$ & $\sim \mathrm{E} 18.5-\mathrm{P} 4$ & $\begin{array}{c}\text { Not yet } \\
\text { expressed }\end{array}$ & $\begin{array}{c}\text { Not yet } \\
\text { expressed }\end{array}$ \\
\hline \multicolumn{9}{|c|}{$\begin{array}{l}\text { ND, not determined. Super., superficial sublayer. P: postnatal; E: embryonic. Adult: } 2-4 \text { months old. }{ }^{\natural} \text { Dentate gyrus outer granular layer and inner } \\
\text { granular layer. }{ }^{\dagger} \text { CA3 deep-superficial distinction in ciliation and neuronal maturation is not as obvious as CA1. }{ }^{?} \text { Require further verification. }{ }^{\ddagger} \text { CA3 } \\
\text { pyramidal neurons mature several days earlier than CA1 neurons. }{ }^{\#} \text { Based on our immunostaining data using anti-NeuN antibody. }{ }^{f} \text { Outmost } \\
\text { superficial layer in the neocortex }{ }^{*} \text { Based on ISH data from the Allen Brain Atlas Developing Mouse Brain. }\end{array}$} \\
\hline
\end{tabular}




\section{References}

1. Bishop, G. A., Berbari, N. F., Lewis, J., and Mykytyn, K. (2007) Type III adenylyl cyclase localizes to primary cilia throughout the adult mouse brain. J Comp Neurol 505, 562-571

2. Sterpka, A., and Chen, X. (2018) Neuronal and astrocytic primary cilia in the mature brain. Pharmacol Res 137, 114-121

3. Singla, V., and Reiter, J. F. (2006) The primary cilium as the cell's antenna: signaling at a sensory organelle. Science 313, 629-633

4. Valente, E. M., Rosti, R. O., Gibbs, E., and Gleeson, J. G. (2014) Primary cilia in neurodevelopmental disorders. Nat Rev Neurol 10, 27-36

5. Lee, J. E., and Gleeson, J. G. (2011) Cilia in the nervous system: linking cilia function and neurodevelopmental disorders. Curr Opin Neurol 24, 98-105

6. Guemez-Gamboa, A., Coufal, N. G., and Gleeson, J. G. (2014) Primary cilia in the developing and mature brain. Neuron 82, 511-521

7. Lee, J. H., and Gleeson, J. G. (2010) The role of primary cilia in neuronal function. Neurobiol Dis 38, $167-172$

8. Houlden, H., Johnson, J., Gardner-Thorpe, C., Lashley, T., Hernandez, D., Worth, P., Singleton, A. B., Hilton, D. A., Holton, J., Revesz, T., Davis, M. B., Giunti, P., and Wood, N. W. (2007) Mutations in TTBK2, encoding a kinase implicated in tau phosphorylation, segregate with spinocerebellar ataxia type 11. Nat Genet 39, 1434-1436

9. Goetz, S. C., Liem, K. F., Jr., and Anderson, K. V. (2012) The spinocerebellar ataxia-associated gene Tau tubulin kinase 2 controls the initiation of ciliogenesis. Cell 151, 847-858

10. Bowie, E., Norris, R., Anderson, K. V., and Goetz, S. C. (2018) Spinocerebellar ataxia type 11associated alleles of Ttbk2 dominantly interfere with ciliogenesis and cilium stability. PLoS Genet 14, e1007844

11. Ikeuchi, Y., de la Torre-Ubieta, L., Matsuda, T., Steen, H., Okazawa, H., and Bonni, A. (2013) The XLID protein PQBP1 and the GTPase Dynamin 2 define a signaling link that orchestrates ciliary morphogenesis in postmitotic neurons. Cell Rep 4, 879-889

12. Hasenpusch-Theil, K., and Theil, T. (2021) The Multifaceted Roles of Primary Cilia in the Development of the Cerebral Cortex. Front Cell Dev Biol 9, 630161

13. Park, S. M., Jang, H. J., and Lee, J. H. (2019) Roles of Primary Cilia in the Developing Brain. Front Cell Neurosci 13, 218

14. Ruat, M., Roudaut, H., Ferent, J., and Traiffort, E. (2012) Hedgehog trafficking, cilia and brain functions. Differentiation 83, S97-104

15. Bangs, F., and Anderson, K. V. (2017) Primary Cilia and Mammalian Hedgehog Signaling. Cold Spring Harb Perspect Biol 9

16. Pal, K., and Mukhopadhyay, S. (2015) Primary cilium and sonic hedgehog signaling during neural tube patterning: role of GPCRs and second messengers. Dev Neurobiol 75, 337-348

17. Mukhopadhyay, S., Wen, X., Ratti, N., Loktev, A., Rangell, L., Scales, S. J., and Jackson, P. K. (2013) The ciliary G-protein-coupled receptor Gpr161 negatively regulates the Sonic hedgehog pathway via cAMP signaling. Cell 152, 210-223

18. Dessaud, E., McMahon, A. P., and Briscoe, J. (2008) Pattern formation in the vertebrate neural tube: a sonic hedgehog morphogen-regulated transcriptional network. Development 135, 24892503

19. Gorivodsky, M., Mukhopadhyay, M., Wilsch-Braeuninger, M., Phillips, M., Teufel, A., Kim, C., Malik, N., Huttner, W., and Westphal, H. (2009) Intraflagellar transport protein 172 is essential 
for primary cilia formation and plays a vital role in patterning the mammalian brain. Dev Biol 325, 24-32

20. Higginbotham, H., Guo, J., Yokota, Y., Umberger, N. L., Su, C. Y., Li, J., Verma, N., Hirt, J., Ghukasyan, V., Caspary, T., and Anton, E. S. (2013) Arl13b-regulated cilia activities are essential for polarized radial glial scaffold formation. Nat Neurosci 16, 1000-1007

21. Wechsler-Reya, R. J., and Scott, M. P. (1999) Control of neuronal precursor proliferation in the cerebellum by Sonic Hedgehog. Neuron 22, 103-114

22. Spassky, N., Han, Y. G., Aguilar, A., Strehl, L., Besse, L., Laclef, C., Ros, M. R., Garcia-Verdugo, J. M., and Alvarez-Buylla, A. (2008) Primary cilia are required for cerebellar development and Shh-dependent expansion of progenitor pool. Dev Biol 317, 246-259

23. Chizhikov, V. V., Davenport, J., Zhang, Q., Shih, E. K., Cabello, O. A., Fuchs, J. L., Yoder, B. K., and Millen, K. J. (2007) Cilia proteins control cerebellar morphogenesis by promoting expansion of the granule progenitor pool. J Neurosci 27, 9780-9789

24. Amador-Arjona, A., Elliott, J., Miller, A., Ginbey, A., Pazour, G. J., Enikolopov, G., Roberts, A. J., and Terskikh, A. V. (2011) Primary cilia regulate proliferation of amplifying progenitors in adult hippocampus: implications for learning and memory. $J$ Neurosci 31, 9933-9944

25. Breunig, J. J., Sarkisian, M. R., Arellano, J. I., Morozov, Y. M., Ayoub, A. E., Sojitra, S., Wang, B., Flavell, R. A., Rakic, P., and Town, T. (2008) Primary cilia regulate hippocampal neurogenesis by mediating sonic hedgehog signaling. Proc Natl Acad Sci U S A 105, 13127-13132

26. Berbari, N. F., Malarkey, E. B., Yazdi, S. M., McNair, A. D., Kippe, J. M., Croyle, M. J., Kraft, T. W., and Yoder, B. K. (2014) Hippocampal and cortical primary cilia are required for aversive memory in mice. PLoS One 9, e106576

27. Tsai, L. H., and Gleeson, J. G. (2005) Nucleokinesis in neuronal migration. Neuron 46, 383-388

28. Pocha, S. M., and Montell, D. J. (2014) Cellular and molecular mechanisms of single and collective cell migrations in Drosophila: themes and variations. Annu Rev Genet 48, 295-318

29. Hatten, M. E. (1999) Central nervous system neuronal migration. Annu Rev Neurosci 22, 511-539

30. Marin, O., Valiente, M., Ge, X., and Tsai, L. H. (2010) Guiding neuronal cell migrations. Cold Spring Harb Perspect Biol 2, a001834

31. Marin, O., and Rubenstein, J. L. (2003) Cell migration in the forebrain. Annu Rev Neurosci 26, 441-483

32. Rahimi-Balaei, M., Bergen, H., Kong, J., and Marzban, H. (2018) Neuronal Migration During Development of the Cerebellum. Front Cell Neurosci 12, 484

33. Kuijpers, M., and Hoogenraad, C. C. (2011) Centrosomes, microtubules and neuronal development. Mol Cell Neurosci 48, 349-358

34. Higginbotham, H. R., and Gleeson, J. G. (2007) The centrosome in neuronal development. Trends Neurosci 30, 276-283

35. Rivas, R. J., and Hatten, M. E. (1995) Motility and cytoskeletal organization of migrating cerebellar granule neurons. $J$ Neurosci 15, 981-989

36. Kawauchi, T. (2015) Cellullar insights into cerebral cortical development: focusing on the locomotion mode of neuronal migration. Front Cell Neurosci 9, 394

37. Xie, Z., Sanada, K., Samuels, B. A., Shih, H., and Tsai, L. H. (2003) Serine 732 phosphorylation of FAK by Cdk5 is important for microtubule organization, nuclear movement, and neuronal migration. Cell 114, 469-482

38. Satir, P., and Christensen, S. T. (2007) Overview of structure and function of mammalian cilia. Annu Rev Physiol 69, 377-400

39. Ishikawa, T., Ueno, H., Omori, T., and Kikuchi, K. (2021) Cilia and centrosomes: Ultrastructural and mechanical perspectives. Semin Cell Dev Biol 110, 61-69 
40. Khayyeri, H., Barreto, S., and Lacroix, D. (2015) Primary cilia mechanics affects cell mechanosensation: A computational study. J Theor Biol 379, 38-46

41. Bezares-Calderon, L. A., Berger, J., and Jekely, G. (2020) Diversity of cilia-based mechanosensory systems and their functions in marine animal behaviour. Philos Trans $R$ Soc Lond B Biol Sci 375, 20190376

42. Resnick, A., and Hopfer, U. (2007) Force-response considerations in ciliary mechanosensation. Biophys J 93, 1380-1390

43. Prasad, R. M., Jin, X., and Nauli, S. M. (2014) Sensing a sensor: identifying the mechanosensory function of primary cilia. Biosensors (Basel) 4, 47-62

44. Spasic, M., and Jacobs, C. R. (2017) Primary cilia: Cell and molecular mechanosensors directing whole tissue function. Semin Cell Dev Biol 71, 42-52

45. Shinohara, K., Kawasumi, A., Takamatsu, A., Yoshiba, S., Botilde, Y., Motoyama, N., Reith, W., Durand, B., Shiratori, H., and Hamada, H. (2012) Two rotating cilia in the node cavity are sufficient to break left-right symmetry in the mouse embryo. Nat Commun 3, 622

46. Matsumoto, M., Sawada, M., Garcia-Gonzalez, D., Herranz-Perez, V., Ogino, T., Bang Nguyen, H., Quynh Thai, T., Narita, K., Kumamoto, N., Ugawa, S., Saito, Y., Takeda, S., Kaneko, N., Khodosevich, K., Monyer, H., Garcia-Verdugo, J. M., Ohno, N., and Sawamoto, K. (2019) Dynamic Changes in Ultrastructure of the Primary Cilium in Migrating Neuroblasts in the Postnatal Brain. J Neurosci 39, 9967-9988

47. Cembrowski, M. S., Bachman, J. L., Wang, L., Sugino, K., Shields, B. C., and Spruston, N. (2016) Spatial Gene-Expression Gradients Underlie Prominent Heterogeneity of CA1 Pyramidal Neurons. Neuron 89, 351-368

48. Cembrowski, M. S., and Spruston, N. (2019) Heterogeneity within classical cell types is the rule: lessons from hippocampal pyramidal neurons. Nat Rev Neurosci 20, 193-204

49. Soltesz, I., and Losonczy, A. (2018) CA1 pyramidal cell diversity enabling parallel information processing in the hippocampus. Nat Neurosci 21, 484-493

50. Hayashi, K., Kubo, K., Kitazawa, A., and Nakajima, K. (2015) Cellular dynamics of neuronal migration in the hippocampus. Front Neurosci 9, 135

51. Mizuseki, K., Diba, K., Pastalkova, E., and Buzsaki, G. (2011) Hippocampal CA1 pyramidal cells form functionally distinct sublayers. Nat Neurosci 14, 1174-1181

52. Danielson, N. B., Zaremba, J. D., Kaifosh, P., Bowler, J., Ladow, M., and Losonczy, A. (2016) Sublayer-Specific Coding Dynamics during Spatial Navigation and Learning in Hippocampal Area CA1. Neuron 91, 652-665

53. Khalaf-Nazzal, R., and Francis, F. (2013) Hippocampal development - old and new findings. Neuroscience 248, 225-242

54. Deguchi, Y., Donato, F., Galimberti, I., Cabuy, E., and Caroni, P. (2011) Temporally matched subpopulations of selectively interconnected principal neurons in the hippocampus. Nat Neurosci 14, 495-504

55. Cooper, J. A. (2008) A mechanism for inside-out lamination in the neocortex. Trends Neurosci 31, 113-119

56. Reiner, O. (2013) LIS1 and DCX: Implications for Brain Development and Human Disease in Relation to Microtubules. Scientifica (Cairo) 2013, 393975

57. Silva, C. G., Peyre, E., and Nguyen, L. (2019) Cell migration promotes dynamic cellular interactions to control cerebral cortex morphogenesis. Nat Rev Neurosci 20, 318-329

58. Chen, X., Luo, J., Leng, Y., Yang, Y., Zweifel, L. S., Palmiter, R. D., and Storm, D. R. (2016) Ablation of Type III Adenylyl Cyclase in Mice Causes Reduced Neuronal Activity, Altered Sleep Pattern, and Depression-like Phenotypes. Biol Psychiatry 80, 836-848 
59. Ferent, J., Constable, S., Gigante, E. D., Yam, P. T., Mariani, L. E., Legue, E., Liem, K. F., Jr., Caspary, T., and Charron, F. (2019) The Ciliary Protein Arl13b Functions Outside of the Primary Cilium in Shh-Mediated Axon Guidance. Cell Rep 29, 3356-3366 e3353

60. Guo, J., Otis, J. M., Higginbotham, H., Monckton, C., Cheng, J., Asokan, A., Mykytyn, K., Caspary, T., Stuber, G. D., and Anton, E. S. (2017) Primary Cilia Signaling Shapes the Development of Interneuronal Connectivity. Dev Cell 42, 286-300 e284

61. Higginbotham, H., Eom, T. Y., Mariani, L. E., Bachleda, A., Hirt, J., Gukassyan, V., Cusack, C. L., Lai, C., Caspary, T., and Anton, E. S. (2012) Arl13b in primary cilia regulates the migration and placement of interneurons in the developing cerebral cortex. Dev Cell 23, 925-938

62. Li, Y., Tian, X., Ma, M., Jerman, S., Kong, S., Somlo, S., and Sun, Z. (2016) Deletion of ADP Ribosylation Factor-Like GTPase 13B Leads to Kidney Cysts. J Am Soc Nephrol 27, 3628-3638

63. He, K., Ma, X., Xu, T., Li, Y., Hodge, A., Zhang, Q., Torline, J., Huang, Y., Zhao, J., Ling, K., and $\mathrm{Hu}$, J. (2018) Axoneme polyglutamylation regulated by Joubert syndrome protein ARL13B controls ciliary targeting of signaling molecules. Nat Commun 9, 3310

64. Revenkova, E., Liu, Q., Gusella, G. L., and Iomini, C. (2018) The Joubert syndrome protein ARL13B binds tubulin to maintain uniform distribution of proteins along the ciliary membrane. $J$ Cell Sci 131

65. Sterpka, A., Yang, J., Strobel, M., Zhou, Y., Pauplis, C., and Chen, X. (2020) Diverged morphology changes of astrocytic and neuronal primary cilia under reactive insults. Mol Brain 13, 28

66. Gigante, E. D., Taylor, M. R., Ivanova, A. A., Kahn, R. A., and Caspary, T. (2020) ARL13B regulates Sonic hedgehog signaling from outside primary cilia. Elife $\mathbf{9}$

67. Larkins, C. E., Aviles, G. D., East, M. P., Kahn, R. A., and Caspary, T. (2011) Arl13b regulates ciliogenesis and the dynamic localization of Shh signaling proteins. Mol Biol Cell 22, 4694-4703

68. Bangs, F. K., Schrode, N., Hadjantonakis, A. K., and Anderson, K. V. (2015) Lineage specificity of primary cilia in the mouse embryo. Nat Cell Biol 17, 113-122

69. Higginbotham, H., Bielas, S., Tanaka, T., and Gleeson, J. G. (2004) Transgenic mouse line with green-fluorescent protein-labeled Centrin 2 allows visualization of the centrosome in living cells. Transgenic Res 13, 155-164

70. Seixas, C., Choi, S. Y., Polgar, N., Umberger, N. L., East, M. P., Zuo, X., Moreiras, H., Ghossoub, R., Benmerah, A., Kahn, R. A., Fogelgren, B., Caspary, T., Lipschutz, J. H., and Barral, D. C. (2016) Arl13b and the exocyst interact synergistically in ciliogenesis. Mol Biol Cell 27, 308-320

71. Fiala, J. C., Feinberg, M., Popov, V., and Harris, K. M. (1998) Synaptogenesis via dendritic filopodia in developing hippocampal area CA1. J Neurosci 18, 8900-8911

72. Lauri, S. E., Vesikansa, A., Segerstrale, M., Collingridge, G. L., Isaac, J. T., and Taira, T. (2006) Functional maturation of CA1 synapses involves activity-dependent loss of tonic kainate receptormediated inhibition of glutamate release. Neuron 50, 415-429

73. Thakar, S., Wang, L., Yu, T., Ye, M., Onishi, K., Scott, J., Qi, J., Fernandes, C., Han, X., Yates, J. R., 3rd, Berg, D. K., and Zou, Y. (2017) Evidence for opposing roles of Celsr3 and Vangl2 in glutamatergic synapse formation. Proc Natl Acad Sci U S A 114, E610-E618

74. Hsia, A. Y., Malenka, R. C., and Nicoll, R. A. (1998) Development of excitatory circuitry in the hippocampus. J Neurophysiol 79, 2013-2024

75. Sipos, E., Komoly, S., and Acs, P. (2018) Quantitative Comparison of Primary Cilia Marker Expression and Length in the Mouse Brain. J Mol Neurosci 64, 397-409

76. Ponnio, T., and Conneely, O. M. (2004) nor-1 regulates hippocampal axon guidance, pyramidal cell survival, and seizure susceptibility. Mol Cell Biol 24, 9070-9078 
77. Valero, M., Cid, E., Averkin, R. G., Aguilar, J., Sanchez-Aguilera, A., Viney, T. J., GomezDominguez, D., Bellistri, E., and de la Prida, L. M. (2015) Determinants of different deep and superficial CA1 pyramidal cell dynamics during sharp-wave ripples. Nat Neurosci 18, 1281-1290

78. Radic, T., Friess, L., Vijikumar, A., Jungenitz, T., Deller, T., and Schwarzacher, S. W. (2017) Differential Postnatal Expression of Neuronal Maturation Markers in the Dentate Gyrus of Mice and Rats. Front Neuroanat 11, 104

79. Sarnat, H. B., Nochlin, D., and Born, D. E. (1998) Neuronal nuclear antigen (NeuN): a marker of neuronal maturation in early human fetal nervous system. Brain Dev 20, 88-94

80. Gusel'nikova, V. V., and Korzhevskiy, D. E. (2015) NeuN As a Neuronal Nuclear Antigen and Neuron Differentiation Marker. Acta Naturae 7, 42-47

81. Reiter, J. F., Blacque, O. E., and Leroux, M. R. (2012) The base of the cilium: roles for transition fibres and the transition zone in ciliary formation, maintenance and compartmentalization. $E M B O$ Rep 13, 608-618

82. Chen, H. Y., Kelley, R. A., Li, T., and Swaroop, A. (2021) Primary cilia biogenesis and associated retinal ciliopathies. Semin Cell Dev Biol 110, 70-88

83. Kitazawa, A., Kubo, K., Hayashi, K., Matsunaga, Y., Ishii, K., and Nakajima, K. (2014) Hippocampal pyramidal neurons switch from a multipolar migration mode to a novel "climbing" migration mode during development. J Neurosci 34, 1115-1126

84. Kirschen, G. W., Liu, H., Lang, T., Liang, X., Ge, S., and Xiong, Q. (2017) The radial organization of neuronal primary cilia is acutely disrupted by seizure and ischemic brain injury. Front Biol (Beijing) 12, 124-138

85. Angevine, J. B., Jr. (1965) Time of neuron origin in the hippocampal region. An autoradiographic study in the mouse. Exp Neurol Suppl, Suppl 2:1-70

86. Caviness, V. S., Jr. (1973) Time of neuron origin in the hippocampus and dentate gyrus of normal and reeler mutant mice: an autoradiographic analysis. J Comp Neurol 151, 113-120

87. Stanfield, B. B., and Cowan, W. M. (1979) The development of the hippocampus and dentate gyrus in normal and reeler mice. J Comp Neurol 185, 423-459

88. Xie, Z., Ma, X., Ji, W., Zhou, G., Lu, Y., Xiang, Z., Wang, Y. X., Zhang, L., Hu, Y., Ding, Y. Q., and Zhang, W. J. (2010) Zbtb20 is essential for the specification of CA1 field identity in the developing hippocampus. Proc Natl Acad Sci U S A 107, 6510-6515

89. Isaac, J. T., Nicoll, R. A., and Malenka, R. C. (1995) Evidence for silent synapses: implications for the expression of LTP. Neuron 15, 427-434

90. Xiao, M. Y., Wasling, P., Hanse, E., and Gustafsson, B. (2004) Creation of AMPA-silent synapses in the neonatal hippocampus. Nat Neurosci 7, 236-243

91. Ball, G., and Seal, M. L. (2019) Individual variation in longitudinal postnatal development of the primate brain. Brain Struct Funct 224, 1185-1201

92. Stoufflet, J., Chaulet, M., Doulazmi, M., Fouquet, C., Dubacq, C., Metin, C., Schneider-Maunoury, S., Trembleau, A., Vincent, P., and Caille, I. (2020) Primary cilium-dependent cAMP/PKA signaling at the centrosome regulates neuronal migration. Sci Adv 6

93. Carvajal-Gonzalez, J. M., Roman, A. C., and Mlodzik, M. (2016) Positioning of centrioles is a conserved readout of Frizzled planar cell polarity signalling. Nat Commun 7, 11135

94. Chien, Y. H., Srinivasan, S., Keller, R., and Kintner, C. (2018) Mechanical Strain Determines Cilia Length, Motility, and Planar Position in the Left-Right Organizer. Dev Cell 45, 316-330 e314

95. Chen, X., Xia, Z., and Storm, D. R. (2012) Stimulation of electro-olfactogram responses in the main olfactory epithelia by airflow depends on the type 3 adenylyl cyclase. J Neurosci 32, 1576915778 
bioRxiv preprint doi: https://doi.org/10.1101/2021.12.21.473383; this version posted January 18, 2022. The copyright holder for this preprint (which was not certified by peer review) is the author/funder. All rights reserved. No reuse allowed without permission.

Fig. 1
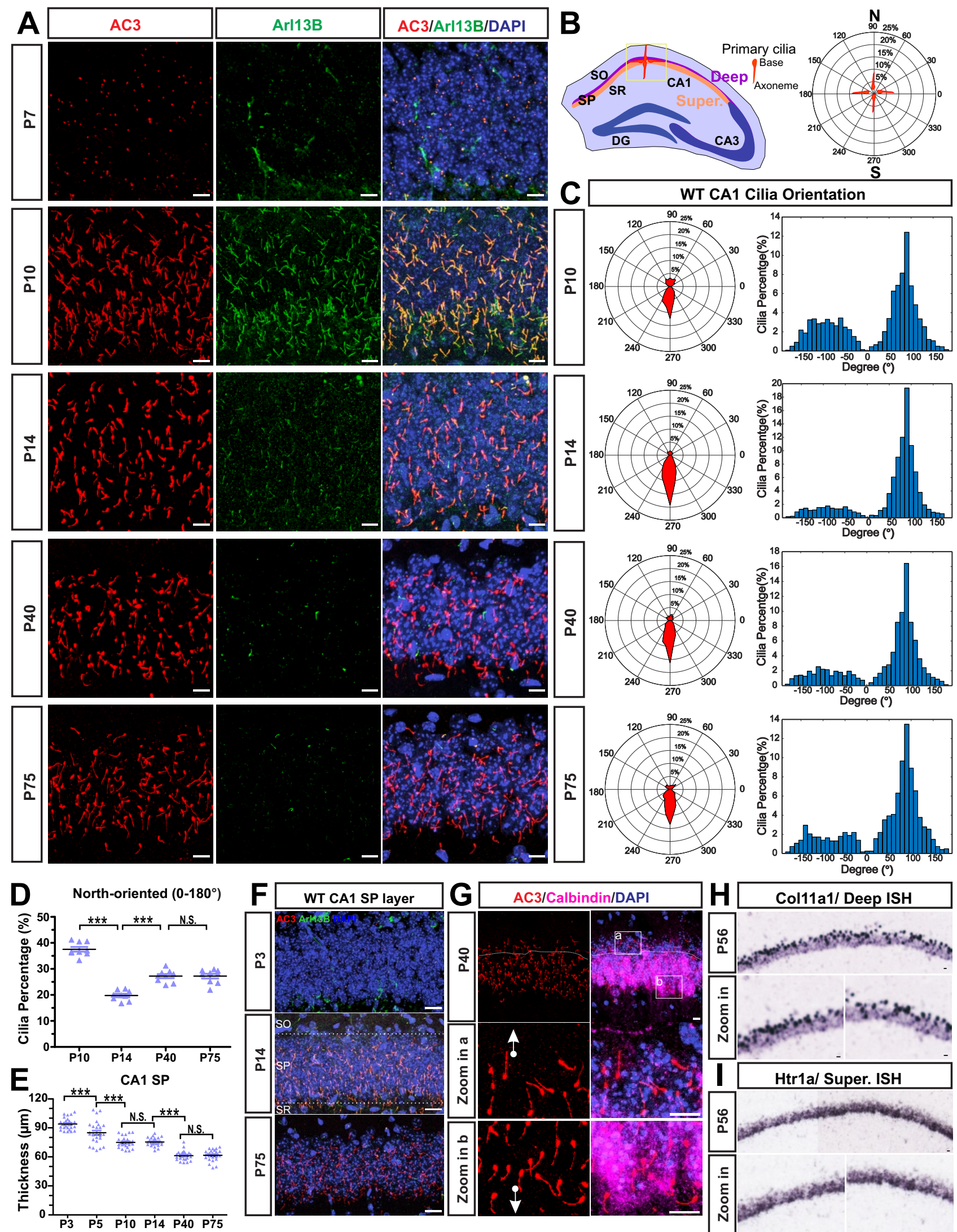
Fig. 2
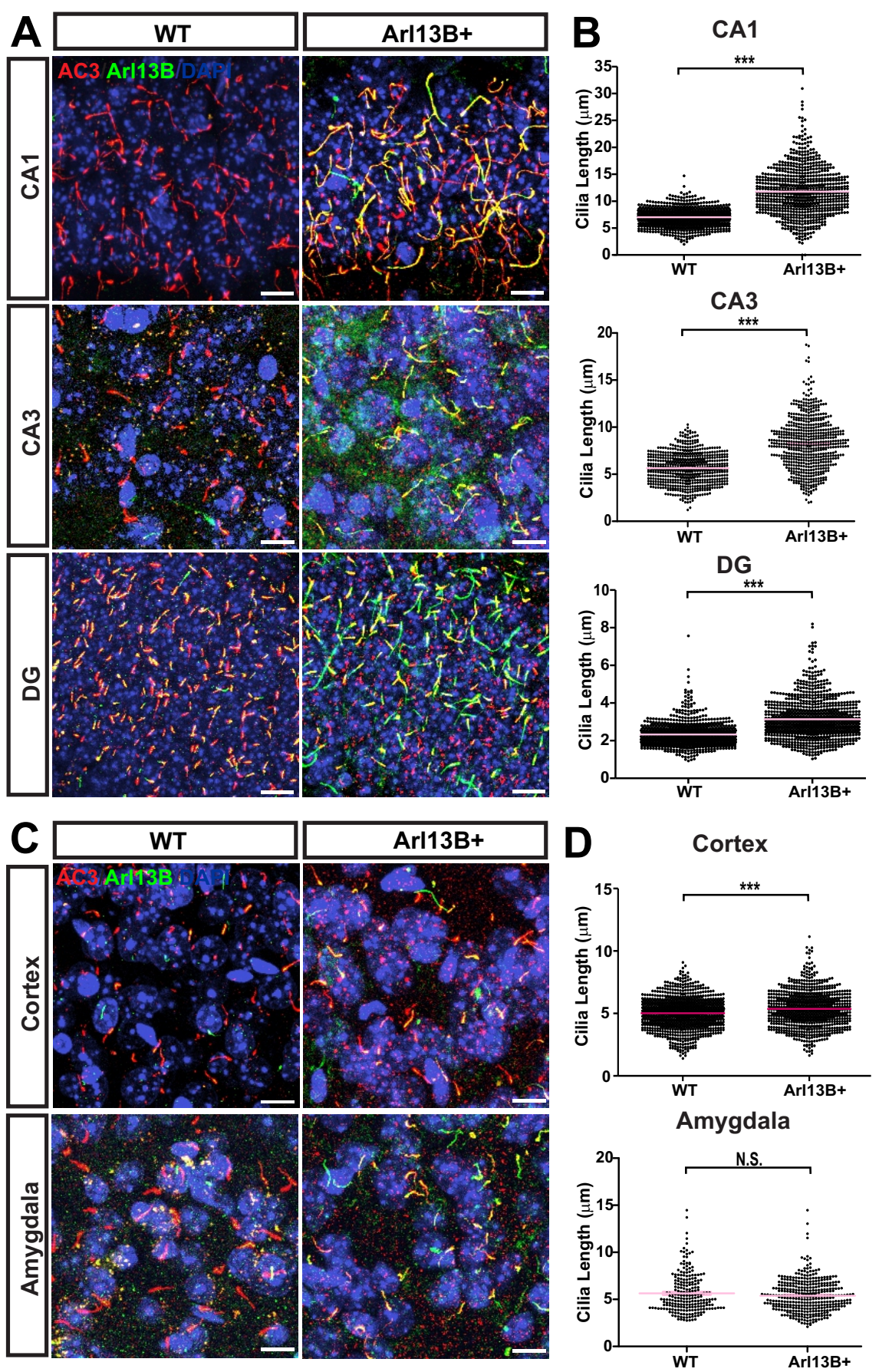
Fig. 3
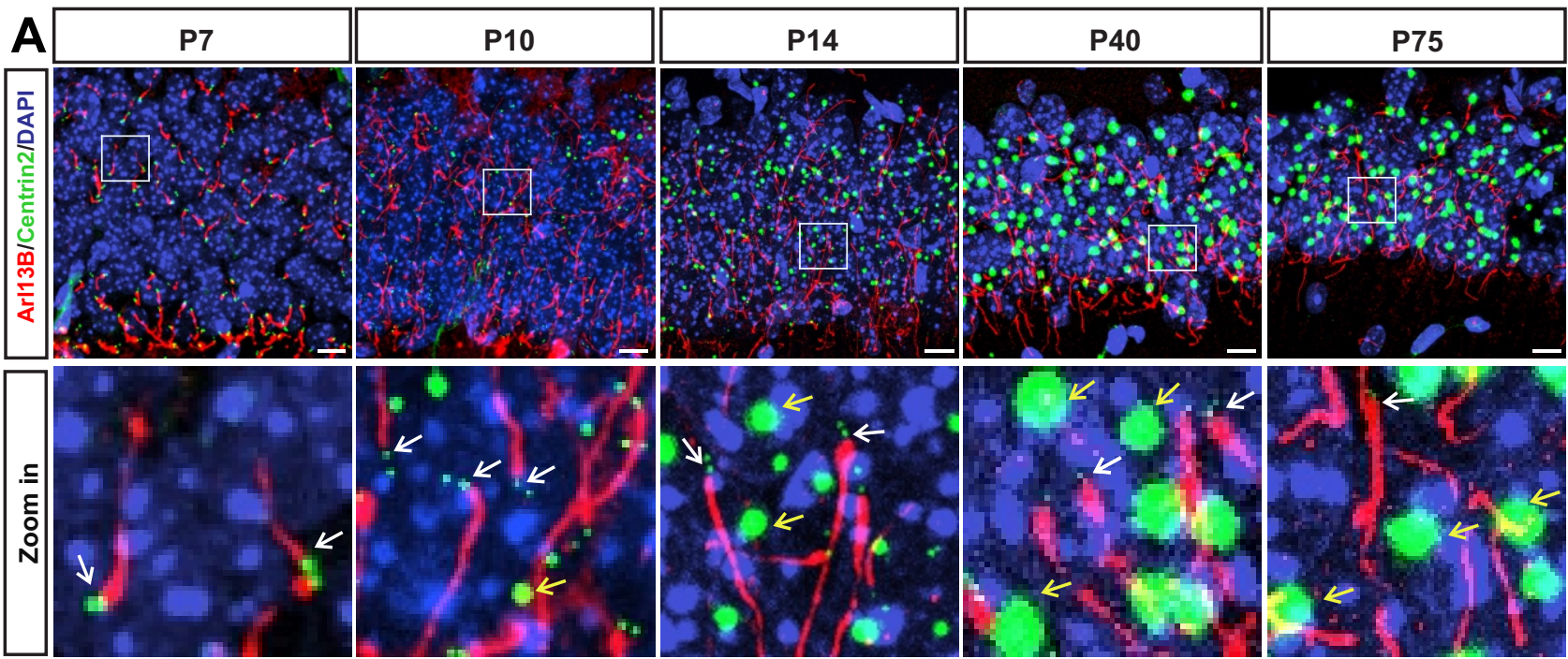

\section{B}
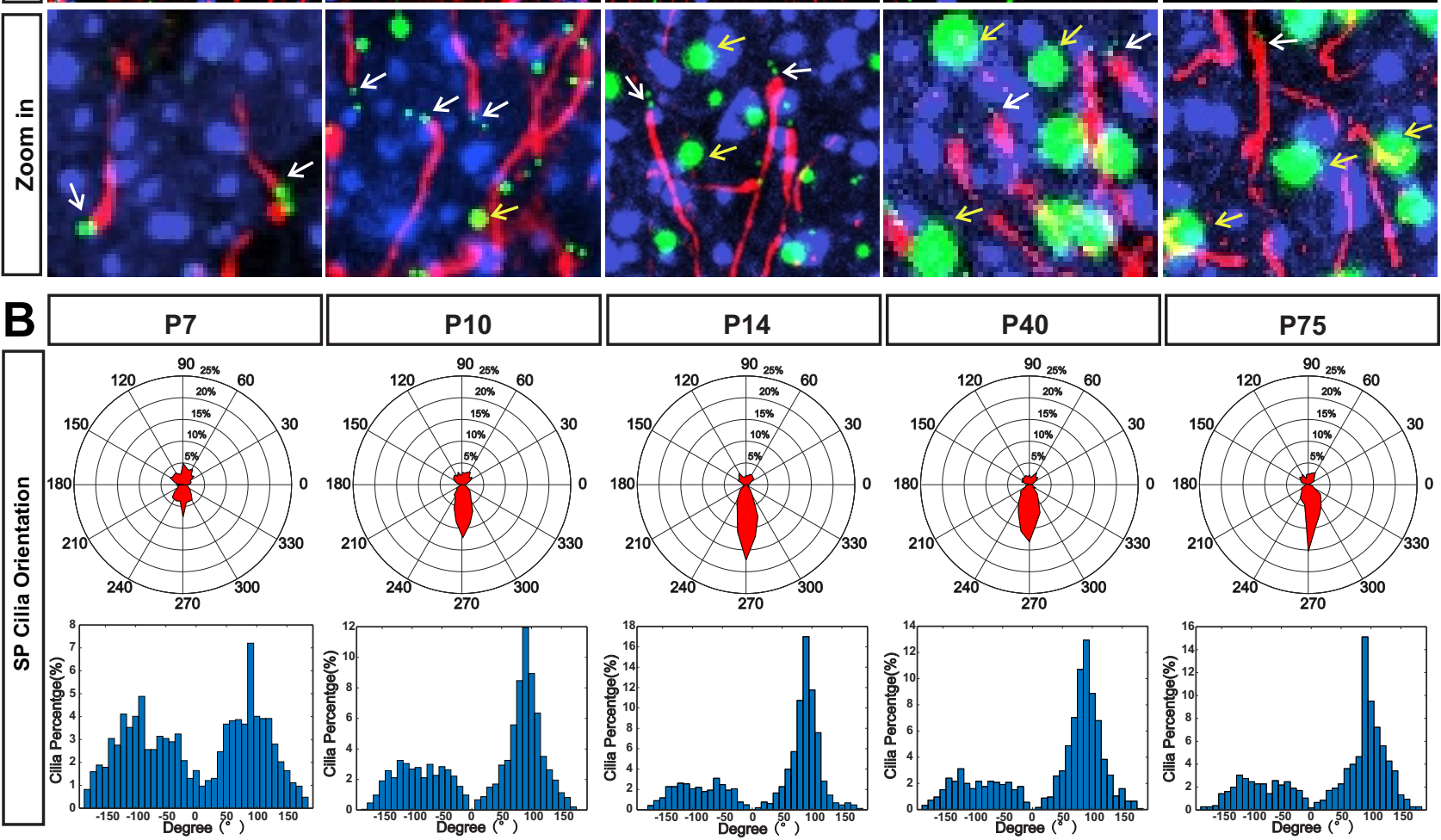

C North-oriented (0-180 $)$

D North-oriented $\left(\mathbf{0 - 1 8 0 ^ { \circ } )}\right.$
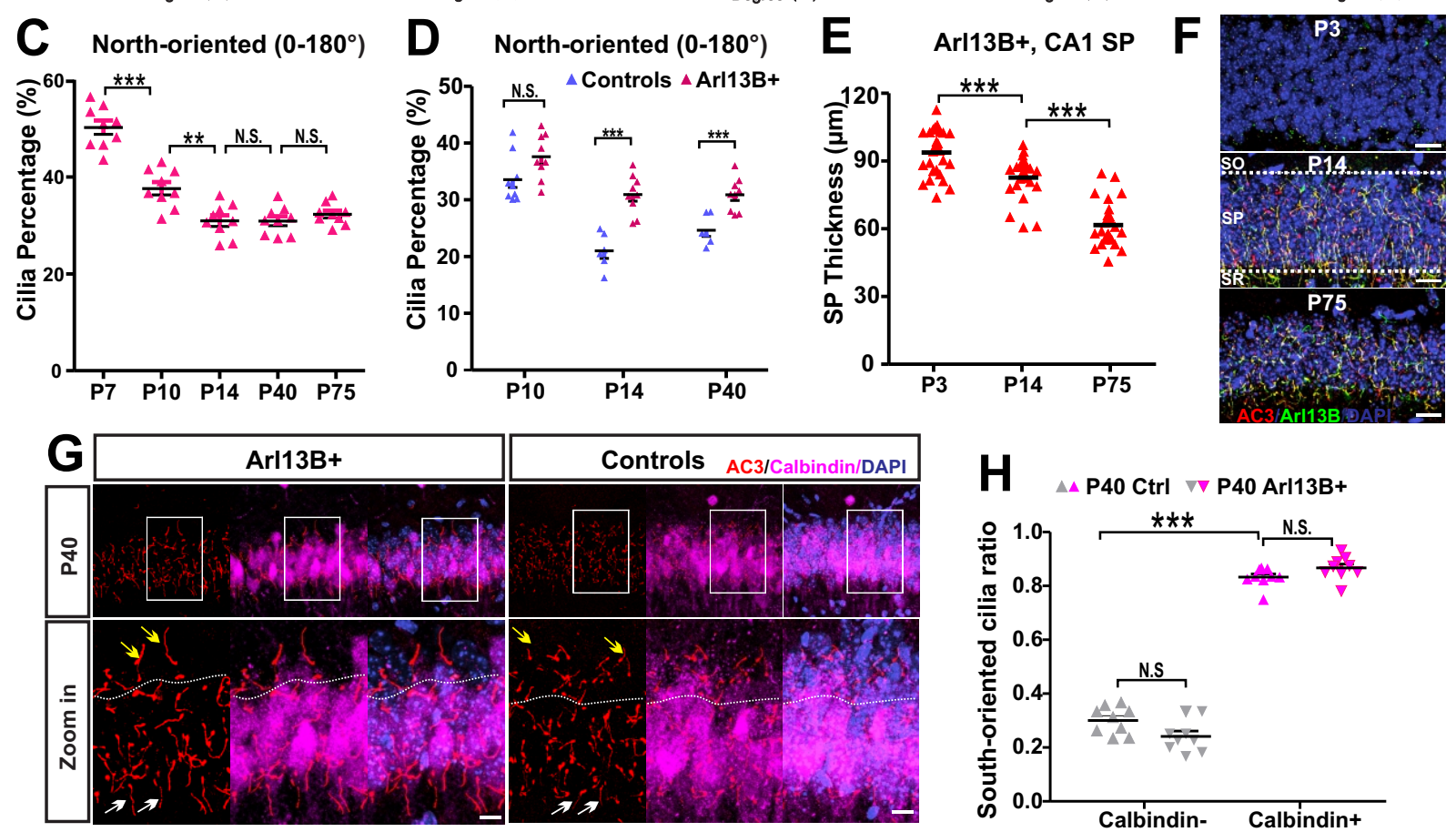

H $\triangle \triangle$ P40 Ctrl $~ ¥ P$ P40 Arl13B+

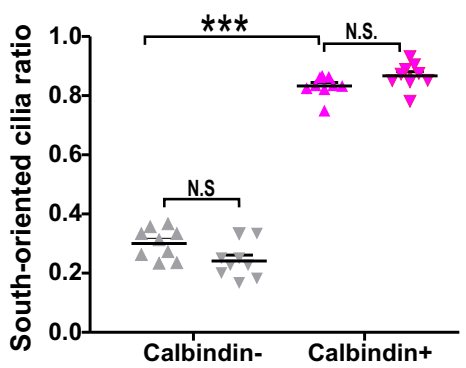


bioRxiv preprint doi: https://doi.org/10.1101/2021.12.21.473383; this version posted January 18, 2022. The copyright holder for this preprint (which was not certified by peer review) is the author/funder. All rights reserved. No reuse allowed without permission.

Fig. 4

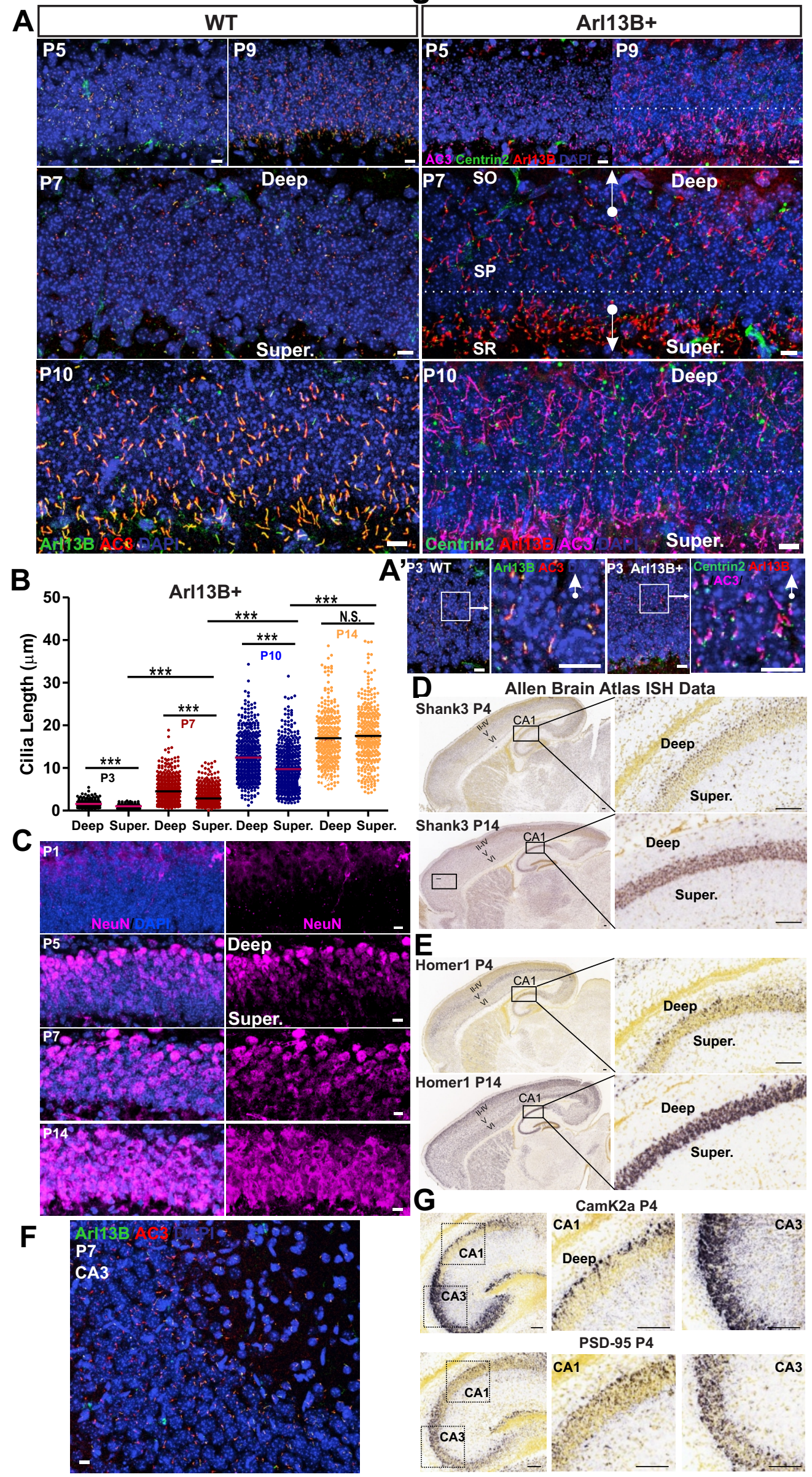


bioRxiv preprint doi: https://doi.org/10.1101/2021.12.21.473383; this version posted January 18, 2022. The copyright holder for this preprint (which was not certified by peer review) is the author/funder. All rights reserved. No reuse allowed without permission.

Fig. 5
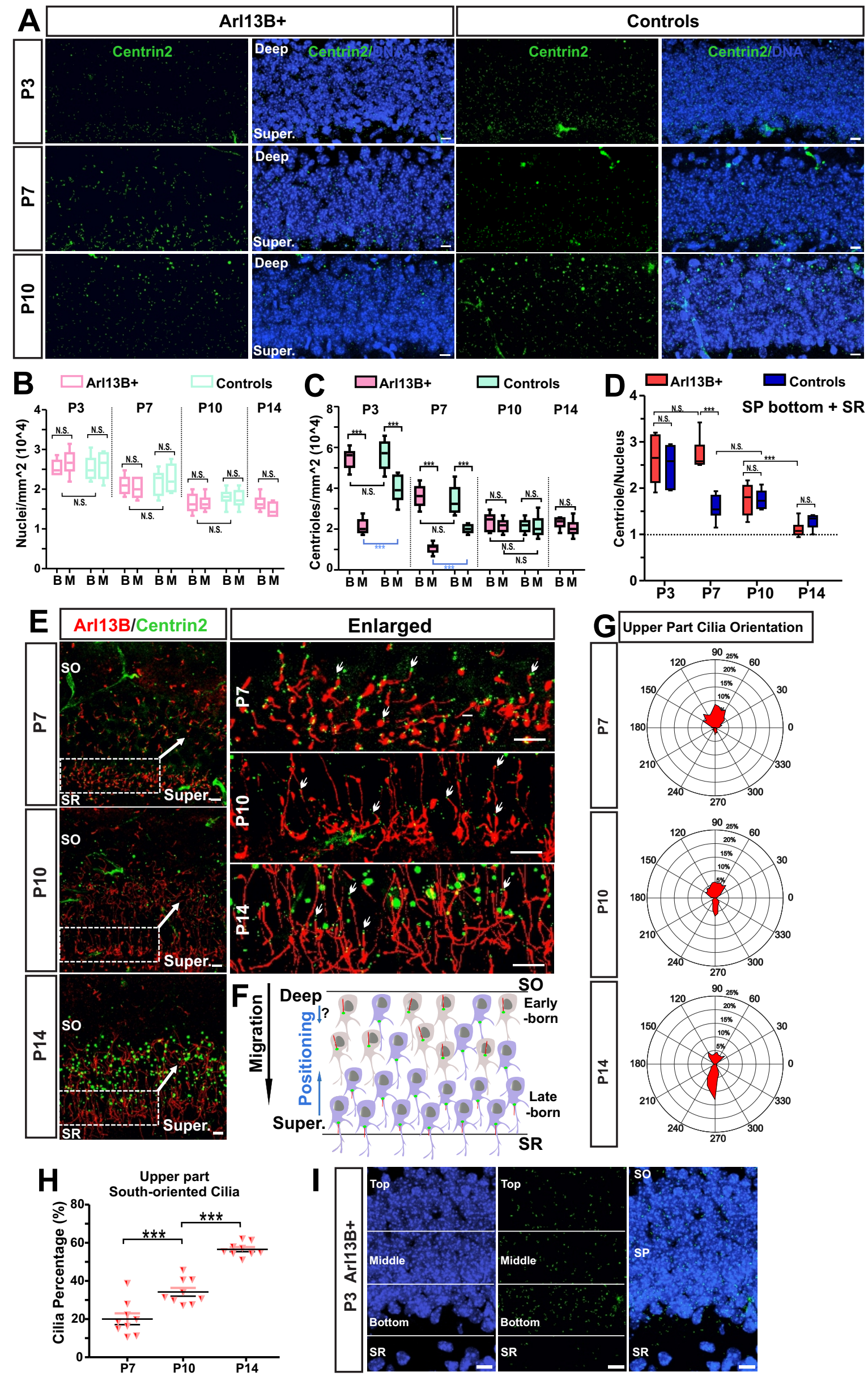
bioRxiv preprint doi: https://doi.org/10.1101/2021.12.21.473383; this version posted January 18, 2022. The copyright holder for this preprint (which was not certified by peer review) is the author/funder. All rights reserved. No reuse allowed without permission.

\section{Fig. 6}

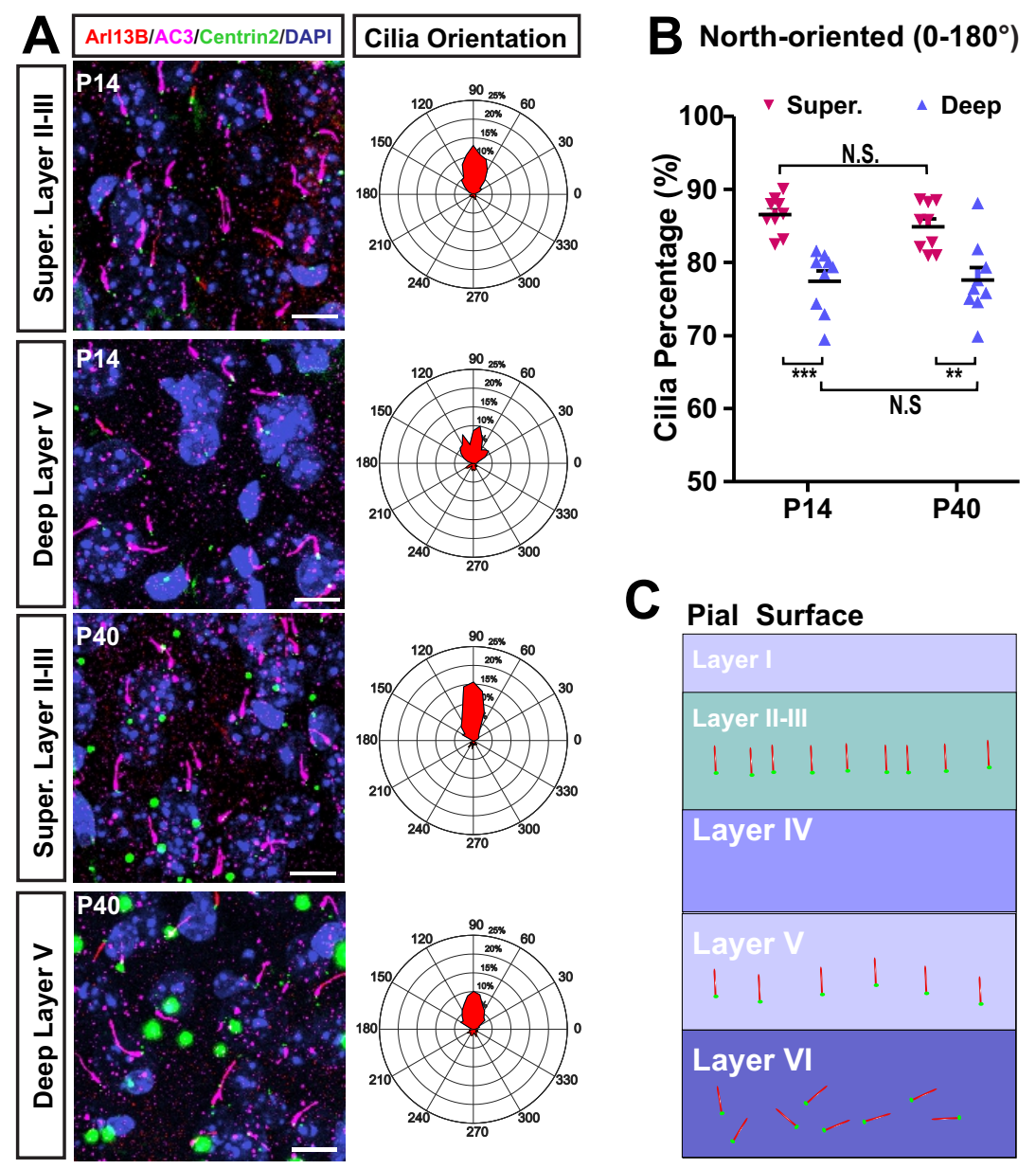


Fig. 7
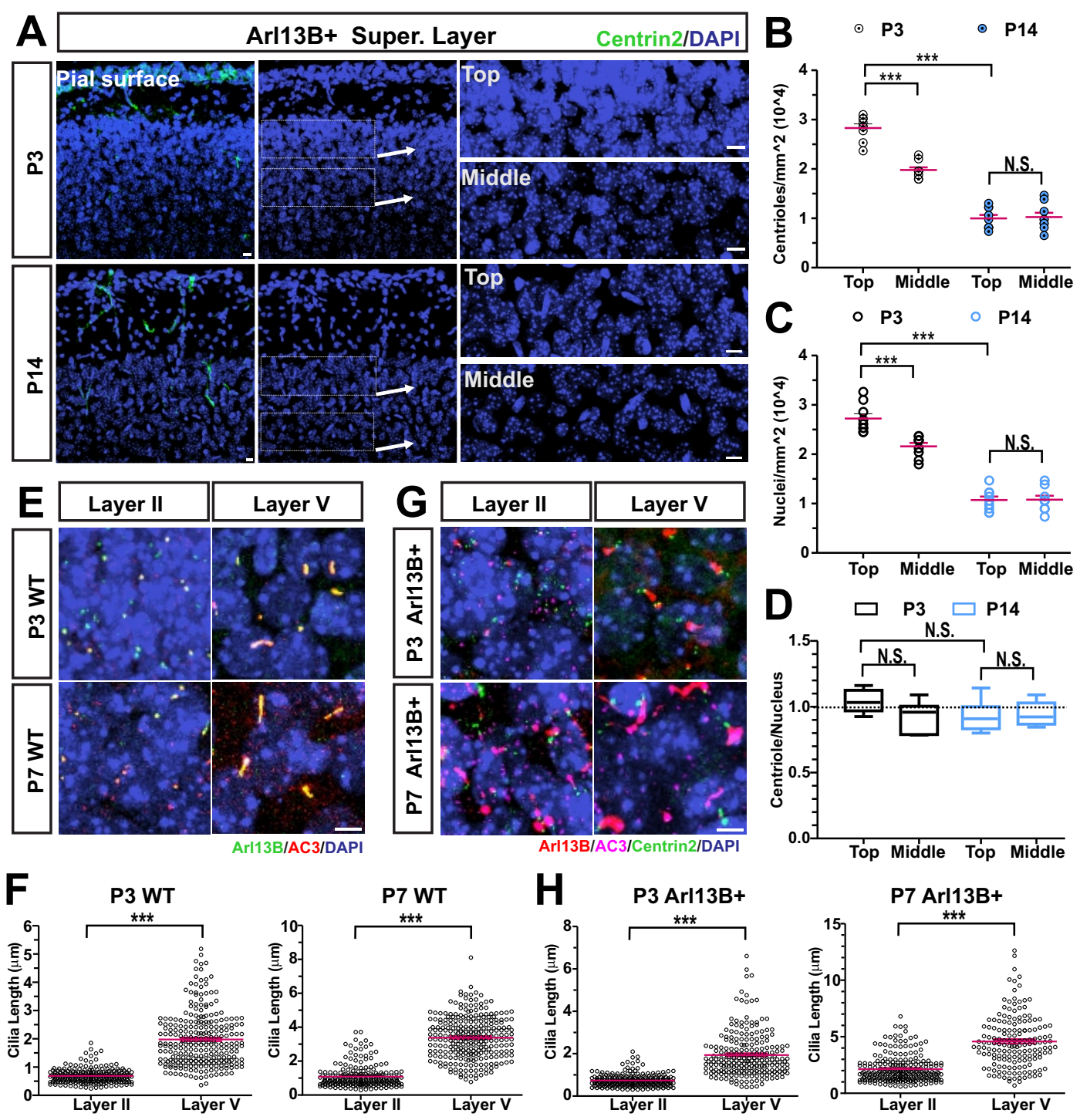